\title{
1 Haplotype-based inference of the distribution of fitness
}

\section{2 effects}

4 Diego Ortega-Del Vecchyo ${ }^{1,2^{*}}$, Kirk E. Lohmueller ${ }^{2,3,4}$ and John Novembre ${ }^{5,6^{*}}$

51 International Laboratory for Human Genome Research, National Autonomous University of

6 Mexico, Santiago de Querétaro, Querétaro, 76230, México.

72 Interdepartmental Program in Bioinformatics, University of California, Los Angeles, CA 90095,

8 United States of America.

93 Department of Ecology and Evolutionary Biology, University of California, Los Angeles, CA

10 90095, United States of America.

114 Department of Human Genetics, David Geffen School of Medicine, University of California,

12 Los Angeles, CA, 90095, United States of America.

135 Department of Human Genetics, University of Chicago, Chicago, Illinois, 60637, United States

14 of America.

156 Department of Ecology and Evolution, University of Chicago, Chicago, Illinois, 60637, United

16 States of America.

*dortega@liigh.unam.mx (DO-DV); jnovembre@uchicago.edu (JN)

\section{Abstract}

21 Recent genome sequencing studies with large sample sizes in humans have discovered a vast

22 quantity of low-frequency variants, providing an important source of information to analyze how

23 selection is acting on human genetic variation. In order to estimate the strength of natural

24 selection acting on low-frequency variants, we have developed a likelihood-based method that

25 uses the lengths of pairwise identity-by-state between haplotypes carrying low-frequency

26 variants. We show that in some non-equilibrium populations (such as those that have had

27 recent population expansions) it is possible to distinguish between positive or negative selection

28 acting on a set of variants. With our new framework, one can infer a fixed selection intensity

29 acting on a set of variants at a particular frequency, or a distribution of selection coefficients for

30 standing variants and new mutations. We apply our method to the UK1OK phased haplotype

31 dataset of 3,781 individuals and find a similar proportion of neutral, moderately deleterious, and

32 deleterious variants compared to previous estimates made using the site frequency spectrum. 
33 We discuss several interpretations for this result, including that selective constraints have

34 remained constant over time.

\section{Introduction}

37 The distribution of fitness effects for new mutations $(D F E)$ is one of the most important 38 determinants of molecular evolution. The $D F E$ is a probability distribution that quantifies the 39 proportion of new mutations having a certain selection coefficient $s$, where $s$ can take positive or 40 negative values depending on whether the allele is under positive or negative selection. The 41 DFE determines current levels of genetic variation, since the frequencies of the alleles under 42 selection depend on their selection coefficient (Sawyer \& Hartl 1992; Hartl et al. 1994; 43 Bustamante et al. 2001), and alleles under selection change the genetic variation at linked sites 44 due to the effects of linked selection (Maynard Smith \& Haigh 1974; Charlesworth et al. 1993). 45 The $D F E$ is also a key feature in the evolution of complex phenotypic traits (Lohmueller 2014a; 46 Simons et al. 2014; Mancuso et al. 2015), since the association between the selection 47 coefficients and the effect of mutations on a complex trait is an important determinant of the 48 genetic architecture of a trait (Eyre-Walker 2010). Due to the impact of the DFE on levels of 49 genetic and phenotypic variation, properly inferring the $D F E$ is essential to many fundamental 50 problems such as validating predictions of the nearly neutral theory (Kimura \& Crow 1964; Crow 51 1972; Ohta 1992), understanding changes in the deleterious segregating variation observed in 52 different populations (Gazave et al. 2013; Lohmueller 2014b; Henn et al. 2015; Brandvain \& 53 Wright 2016; Gravel 2016; Simons \& Sella 2016; Koch \& Novembre 2017), elucidating the

54 factors that influence changes on the DFE between species (Martin \& Lenormand 2006; 55 Charlesworth \& Eyre-Walker 2007; Serohijos \& Shakhnovich 2014; Tenaillon 2014; Rice et al. 56 2015; Huber et al. 2017), and inferring the amount of adaptive evolution between species 57 (Gossmann et al. 2012; Galtier 2016; Zhen et al. 2018).

58 Broadly, two lines of research have been developed to infer a DFE. One is based on 59 experimental approaches and the other one is based on the analysis of population genetic 60 variation at putatively neutral and deleterious sites. The main experimental approaches taken 61 with viruses, bacteria and yeast are site-directed mutagenesis experiments in target regions 62 (Bataillon \& Bailey 2014) and mutation-accumulation experiments (Halligan \& Keightley 2009).

63 They are useful because they can obtain information about the DFE including advantageous and 64 deleterious mutations; that said, advantageous mutations tend to be rare or not found in results 65 from experimental approaches (Halligan \& Keightley 2009; Lind et al. 2010; Jacquier et al. 2013; 
Bataillon \& Bailey 2014) with some exceptions (Sanjuán et al. 2004; Dickinson 2008). The types of probability distributions that have provided a good fit to the DFE of deleterious mutations on site-directed mutagenesis experiments are a gamma distribution (Domingo-Calap et al. 2009; Lind et al. 2010; Jacquier et al. 2013), a unimodal distribution with a similar shape to a gamma distribution (Sanjuán et al. 2004; Domingo-Calap et al. 2009; Peris et al. 2010), and a bimodal distribution with one part of the probability mass on nearly neutral mutations and the other one on the highly deleterious mutations (Hietpas et al. 2011). However, the data still points to a bimodal DFE with mutations being either neutral or very deleterious in the majority of the studies where other unimodal simpler distributions provided the best fit to the data (Sanjuán et al. 2004; Domingo-Calap et al. 2009; Peris et al. 2010; Jacquier et al. 2013). This highlights that the DFE might have a more complex form than the simpler probability distributions typically used to fit data. In mutation-accumulation experiments, a gamma distribution is typically assumed for the $D F E$ of deleterious mutations, since there is little information to distinguish between alternative distributions (Halligan \& Keightley 2009).

The other main approach is to use population genetic variation data to estimate the $D F E$ with information from the site frequency spectrum $(S F S)$ on putatively neutral and deleterious

82 sites (Sawyer \& Hartl 1992; Williamson et al. 2005; Keightley \& Eyre-Walker 2007; Boyko et al.

83 2008; Gutenkunst et al. 2009; Kim et al. 2017). An interesting extension has recently been

84 developed to take SFS information and divergence data from an outgroup to infer the $D F E$ from 85 the population where the SFS data was taken along with the rate of adaptive molecular evolution 86 based on the divergence data (Tataru et al. 2017). Two other extensions have been taken to 87 model the correlation between the fitness effects of multiple nonsynonymous alleles at a 88 particular position (Ragsdale et al. 2016) and to calculate the joint DFE between pairs of 89 populations (Fortier et al. 2019). The first step in these approaches is to inter the demographic 90 scenario that fits the SFS at putatively neutral sites, which typically are chosen to be variants at 91 synonymous sites. The $D F E$ is then inferred from putatively deleterious sites of interest, typically 92 nonsynonymous sites, while taking the demographic scenario into account. Some species 93 where these approaches have been applied to infer the DFE include humans (Eyre-Walker et al. 94 2006; Boyko et al. 2008; Li et al. 2010; Huber et al. 2017; Kim et al. 2017), mouse 95 (Kousathanas \& Keightley 2013; Halligan et al. 2013) and Drosophila (Kousathanas \& Keightley 96 2013; Huber et al. 2017). Studies that compare the fit of different probability distributions argue 97 in favor of a $D F E$ of deleterious nonsynonymous mutations on humans that follows either 1) a 98 gamma distribution (Boyko et al. 2008; Kim et al. 2017) or 2) a combination of a point mass at 99 neutrality plus a gamma distribution (Kim et al. 2017). Those two studies infer a leptokurtic DFE 
100 with a proportion of nearly neutral mutations $\left(s<10^{-5}\right)$ of $18.3 \%-26.3 \%$, and moderate to strong 101 deleterious mutations $\left(s>10^{-3}\right)$ of $46.6 \%-57.4 \%$.

$102 \quad$ One drawback of current methods that estimate the $D F E$ using population genetic 103 variation is that they ignore all linkage information. No attempt has been made to exploit the 104 information from linked genetic variation to estimate the DFE despite the fact that many studies 105 have analyzed how both deleterious (Charlesworth et al. 1993, 1995; Hudson \& Kaplan 1995; 106 Nordborg et al. 1996; Nicolaisen \& Desai 2013; Cvijović et al. 2018) and advantageous variants 107 (Maynard Smith \& Haigh 1974; Kaplan et al. 1989; Braverman et al. 1995; Nielsen 2005) 108 decrease linked genetic variation. Further, linked genetic variation has been effectively used to 109 infer the age of particular variants (Slatkin \& Rannala 1997; Tishkoff et al. 2007; Chen \& Slatkin 110 2013; Mathieson \& McVean 2014; Chen et al. 2015; Nakagome et al. 2016; Ormond et al. 2016; 111 Albers \& McVean 2018), the time to the common ancestor of a positively selected allele (Smith 112 et al. 2018), the time since fixation of an advantageous allele (Przeworski 2003), the selection 113 coefficient of an allele (Slatkin 2001, 2008; Coop \& Griffiths 2004; Tishkoff et al. 2007; Chen \& 114 Slatkin 2013; Chen et al. 2015; Ormond et al. 2016) and to detect loci under positive selection 115 (Kim \& Stephan 2002; Sabeti et al. 2002, 2007; Wang et al. 2006; Voight et al. 2006; Williamson 116 et al. 2007; Tang et al. 2007; Pavlidis et al. 2010; Li 2011; Ferrer-Admetlla et al. 2014; Garud et 117 al. 2015; Field et al. 2016; Huber et al. 2016). Since there has been so much success in 118 understanding how selection changes the linked variation around individual variants, it should 119 be feasible to pool the haplotype information from many variants putatively under selection at a 120 certain frequency $f$ to infer the distribution of fitness effects $D F E_{f}$ of variants at a frequency $f$.

$121 \quad$ Here we propose a new approach to infer $D F E_{f}$. We note that $D F E_{f}$ is different from the 122 distribution of fitness effects of new mutations entering the population, which we call the DFE. 123 Natural selection acts to increase the frequency of advantageous variants and to decrease the 124 frequency of deleterious variants, causing a difference between $D F E$ and $D F E_{f}$. The relationship 125 between $D F E_{f}$ and $D F E$ is one of the topics we will address in this study.

126 Recent large population genomic datasets such as the UK10K (Walter et al. 2015), the 127 Netherlands Genome Project (Francioli et al. 2014) and the Haplotype Reference Consortium 128 (McCarthy et al. 2016) provide an unprecedented source of haplotype information to quantify 129 both the $D F E_{f}$ and the $D F E$. These datasets have started to be exploited to understand the 130 impact of selection on variants under selection at a certain frequency. For example, Kiezun et 131 al. (2013) found that, conditioning on the variants having a certain frequency $f$ in the population, 132 nonsynonymous variants have more extended linkage disequilibrium with neighboring neutral 
133 variation compared to synonymous variants on data from the Netherlands Genome Project. This

134 is in line with Takeo Maruyama's results showing that deleterious variants at a certain frequency

135 have a younger age compared to neutral variants (Maruyama 1974), implying that there is less

136 variation on haplotypes carrying deleterious variants.

$137 \quad$ Building on previous work to estimate the strength of selection acting on advantageous 138 variants (Slatkin 2001; Chen \& Slatkin 2013), we propose an approach to provide a point 139 estimate of the population-scaled selection coefficient or a distribution of fitness effects acting 140 on a set of variants at a particular frequency $f\left(D F E_{f}\right)$. We infer the strength of natural selection 141 using pairwise haplotypic identity-by-state lengths (the length in one direction along a pair of 142 haplotypes carrying a focal allele to the first difference between the pair of haplotypes). For 143 each pair $j$ of haplotypes we define the observed length as $L_{j}$. The length can be measured in 144 both directions along the chromosome extending outward from the focal allele. We show that 145 these lengths can be used to distinguish between alleles under positive and negative selection 146 in several non-equilibrium demographic scenarios. Further, we show how the $D F E_{f}$ can be used 147 to infer the DFE. The resulting method can help improve the understanding of how selection is 148 influencing, for instance, the low-frequency variants present in a population. We apply our 149 method to the UK1OK dataset, and we estimate a similar proportion of neutral, moderately 150 deleterious and deleterious variants compared to SFS-based approaches.

\section{Results}

154 A method for inference of the population-scaled selection coefficient based on haplotype variation

157 Our analysis is based on a set of $x$ haplotype pairs carrying a derived allele at a frequency $f$ in 158 the population. We compute the pairwise identity by state length $L_{j}$ for every haplotype pair, 159 which is defined as the distance from the derived allele to the first difference between a pair of 160 haplotypes. For computational simplicity, we bin the chromosome under analysis into a set of $S$ 161 discrete non-overlapping windows $\boldsymbol{W}=\left\{w_{1}, w_{2}, \ldots, w_{s}\right\}$ that extend to the side of the derived 162 allele. Thus, for a set of $n$ haplotype pairs carrying an allele, our analysis is based on which 163 window the first difference appears in for each pair $\left(\boldsymbol{L}=\left\{L_{1} \in w_{s_{1}}, L_{2} \in w_{s_{2}}, L_{3} \in w_{s_{3}}, \ldots, L_{n} \in\right.\right.$ $\left.\left.164 w_{s_{n}}\right\}\right)$. We define $s_{1}, \ldots, s_{n}$ as integers between 1 and $S$ indicating the windows in which each 
165 length falls (Figure 1). We can calculate a length $L_{j}$ both upstream and downstream of each 166 derived allele in a sample of $n$ allele carriers from alleles at a frequency $f$ in a number $A$ of loci, 167 and observe a total number $x=2 \times A \times\left(\begin{array}{l}n \\ 2\end{array}\right)$ of $\boldsymbol{L}$ length values.

\section{Windows of pairwise haplotypic identity by state lengths (L)}

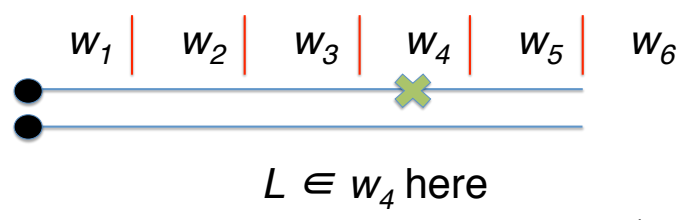

177
Figure 1.- Two haplotypes containing a derived allele, here represented as a black dot, that has a frequency $f$ in the population. The physical distance near the allele is divided into 5 nonoverlapping equidistant windows of a certain length, with an extra window $w_{6}$ indicating that there are no differences in any of the windows $w_{1}$ to $w_{5}$. The first difference between the pairs of haplotypes is denoted by the green " $x$ ". momentarily refer generically to a single observed length as $L$. The parameter we wish to infer is the population scaled selection coefficient $4 N s$. That parameter is defined in terms of the effective population size $N$ from the most ancient epoch in the demographic scenario $D$. It is also possible to define the population scaled selection coefficient in terms of the most recent epoch. coefficient in the most recent time is equal to $4 \frac{N_{R}}{N} s$.

The likelihood of a particular population scaled selection coefficient, $4 \mathrm{Ns}$, conditioned on the allele frequency $f$ and a certain demographic scenario $D$, from a single observed length $L$ can be expressed as:

where $H_{i}$ is a particular allele frequency trajectory. The integration over the space of allele

194 frequency trajectories $H_{i}$ is challenging. One possible approach to do the integration over the 195 space of $H_{i}$ is to perform forward-in-time simulations of alleles under the Poisson Random Field model and retain the trajectories of alleles that end at a frequency $f$ in the present. However,

197 this approach is ineffective because we will end up simulating the trajectories of many alleles 198 that do not end up at a frequency $f$ in the present. To overcome this, we integrate over the 199 space of allele frequency trajectories $H_{i}$ using an importance sampling approach. We also 200 compute $P\left(L \in w_{i} \mid H_{i}\right)$ using a Monte Carlo approximation (see Methods). 
We then apply this likelihood function to the complete collection of observed lengths $\boldsymbol{L}$ to

202 calculate a composite likelihood function for 4 Ns:

An estimator of $4 \mathrm{Ns}$ can be obtained by maximizing this composite likelihood function, which here we do simply by using a grid search over a range of candidate values (see Methods).

To build an understanding of the inference problem and the method's performance, we first assessed the impact of selection on allele frequency trajectories, pairwise coalescent times,

210 and haplotype identity-by-state-lengths, and then assessed the performance of the estimator.

211 We do this first for a constant-size demographic history and then time-varying population sizes.

\section{Evaluation of population-scaled selection coefficient inference for constant population} sizes

We investigated performance using forward-in-time simulations under the Poisson Random

217 Field (PRF) framework. Specifically, we used PReFerSim (Ortega-Del Vecchyo et al. 2016) to 218 obtain 10,000 alleles frequency trajectories with a present-day sample allele frequency of $\hat{p}=1 \%$

219 for 5 different values of selection $(4 N s=0,-50,-100,50,100)$ in a sample of 4,000 chromosomes 220 (see Methods).

Using the 10,000 recorded allele frequency trajectories for each selection value $4 N s$, we

222 calculated the mean allele frequency across many generations going backwards into the past to 223 obtain an average frequency trajectory for $1 \%$ frequency alleles (Figure 2A). As expected, the 224 average allele frequency trajectory for neutral alleles $(4 N s=0)$ is higher for a longer duration 225 going backwards in time compared to alleles under natural selection. Alleles under the same absolute strength of selection have the same average allele frequency trajectory, regardless of whether the allele is under positive or negative selection. The distribution of ages is shifted towards younger values for higher absolute values of $4 N s$ and with increasingly smaller standard deviation (Figure 2B), and Maruyama's theoretical results accurately predict the mean age estimates observed in the simulations (Supplementary Table 1).

231 We computed the distribution of pairwise coalescent times $T_{2}$ analytically (see

232 Supplementary Methods) across different values of $4 \mathrm{Ns}$. We found that alleles under higher

233 absolute values of $4 N s$ have a more recent average value of $T_{2}$, and their distribution of $T_{2}$ has a 234 smaller standard deviation (Figure 2C). We calculated the distribution of $L$ for each $4 N s$ value 
using simulations assuming a constant recombination rate $\rho=4 N r=100$ and a constant mutation rate $\theta=4 N u=100$ for a region of $250 \mathrm{~kb}$. Alleles under the same absolute strength of selection have almost identical distributions of $L$ (Figure 2D). This is in line with the fact that $T_{2}$ is younger in alleles under stronger selection coefficients, implying that there will be fewer mutations between haplotypes sharing the allele and, therefore, higher average values of $L$ (Figure 2E).

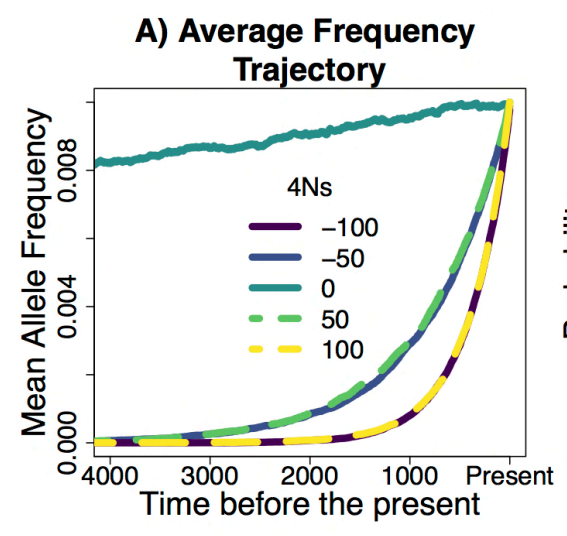

D) Probability Distribution of $L$

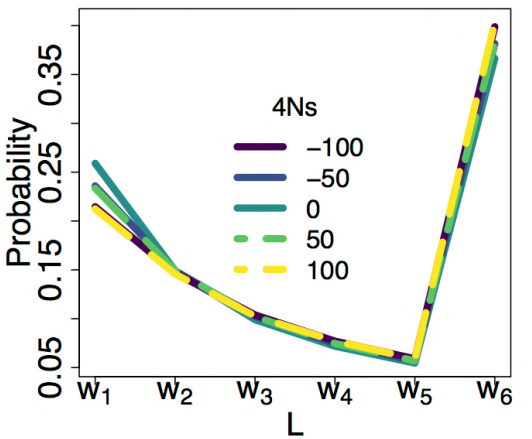

B) Allele Age

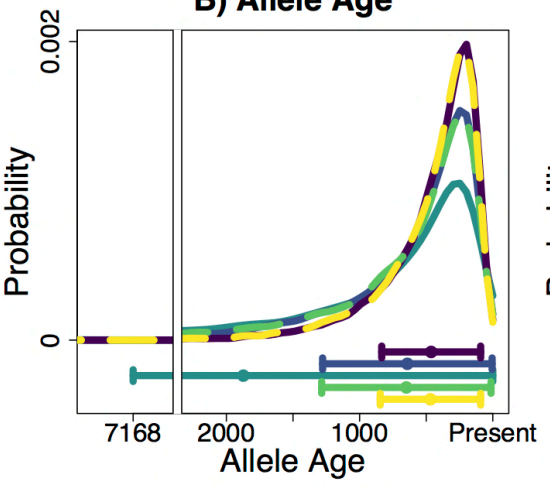

E) The effect of selection on $\mathrm{L}$

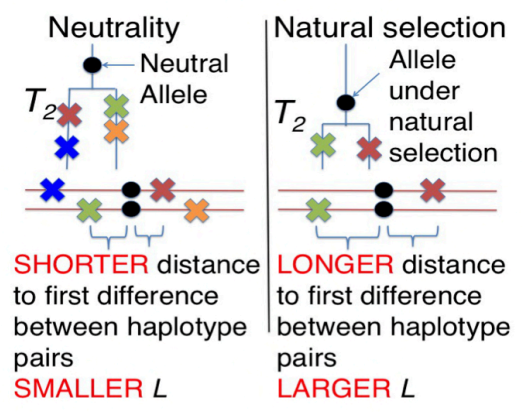

C) $\mathbf{T}_{2}$

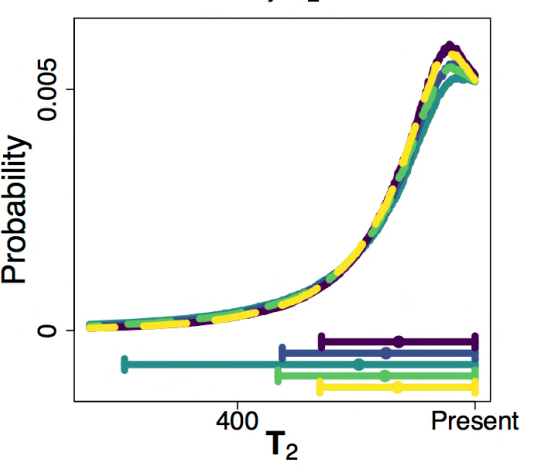

Figure 2.- Properties of alleles sampled at a $1 \%$ frequency under different strengths of natural selection in a constant size population $(\boldsymbol{N}=\mathbf{1 0}, \mathbf{0 0 0})$. We obtained 10,000 frequency trajectories for $1 \%$ frequency alleles under different strengths of selection using forward-in-time simulations under the PRF model. We used those frequency trajectories to calculate: A) The mean allele frequency at different times in the past, in units of generations, to obtain an average frequency trajectory; B) The probability distribution of allele ages; C) The probability distribution of pairwise coalescent times $T_{2}$. Below $B$ ) and $C$ ), we show a dot with two whiskers extending at both sides of the dot. The dot represents the mean value of the distribution and the two whiskers extend one s.d. below or above the mean. The whisker that extends one s.d. below the mean is constrained to extend until max(mean - s.d. ,0). D) Probability distribution of $L$. We define $L$ by taking the physical distance in basepairs next to the allele across 5 non-overlapping equidistant windows of $50 \mathrm{~kb}$, with an extra window $w_{6}$ indicating that there are no differences in the $250 \mathrm{~kb}$ next to the allele. In this demographic scenario, the alleles under a higher absolute strength of selection have younger ages and younger $T_{2}$ on average. The fact that alleles under 
higher strengths of selection have younger average $T_{2}$ values implies that those alleles tend to have larger $L$ values as shown in $\mathrm{D}$ ) and $\mathrm{E}$ ).

We next used the simulations to test our method's ability to estimate the strength of selection. We found that for alleles where, for instance $4 N s$ is -50 , the estimated values of selection tend to be equally distributed around values of -50 or 50 (Figure $3 \mathrm{~A}$ ). A similar result is seen for the $4 N s$ values equal to 100 . This reinforces that in a constant size population one can only provide reasonable estimates of the absolute strength of natural selection. Indeed, when we display the estimated absolute value of the strength of selection, we see that our method produces nearly unbiased estimates (Figure 3B).

A) Inference of Selection

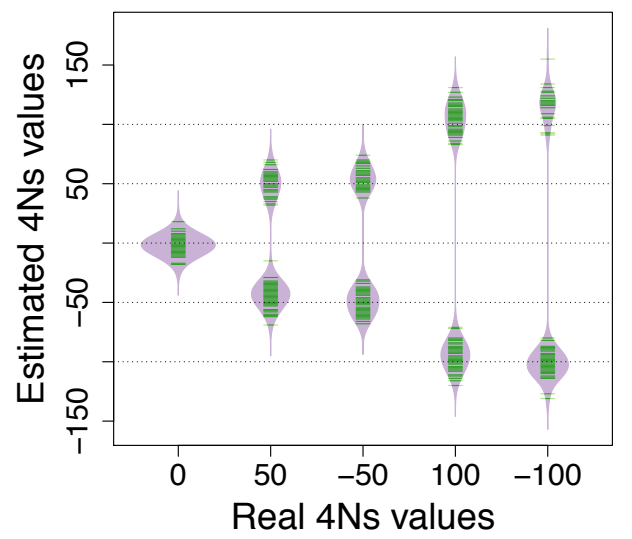

B) Inference of Selection

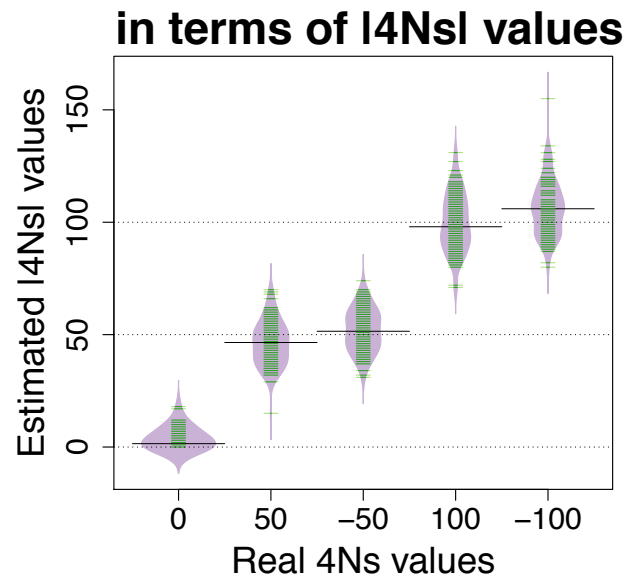

Figure 3.- Estimation of the strength of natural selection in a constant population size model using 10, 000 realized values of $L$ from 10,000 pairs of haplotypes, where each pair was sampled from an independent loci in $1 \%$ frequency alleles. A) Estimated selection values. B) Estimated selection magnitudes (absolute values of $4 N s$ ). 'Real $4 N s$ values' refers to the $4 N s$ values used in the simulations, while 'Estimated $4 N s$ values' refers to the values estimated by our method. The dashed lines are placed on values that match $4 N s$ values used in the simulations. The median value of the estimates of $4 N s$ is shown with a solid line. The green lines in A) and B) indicate estimated values of $4 \mathrm{Ns}$, where there are 100 estimated values for the five $4 N s$ values inspected. Each estimated $4 N s$ value uses 10,000 $L$ values.

\section{Evaluation of inference performance for non-equilibrium demographic scenarios}

Following our analysis for constant-size populations, we next analyzed the shape of the average allele frequency trajectory in a population expansion scenario (Figure $4 \mathrm{~A}$ ) for $1 \%$ frequency alleles with different $4 N s$ values. Unlike in the constant population size scenario, we found distinct average allele frequency trajectories for alleles under positive or negative selection (Figure 4B): alleles under positive selection on average had increased in frequency moving forward in time, while alleles under negative selection on average had increased in frequency 
before the expansion and then decreased after the expansion due to the increased selection efficacy in the large population. The ages of alleles under the strongest absolute values of selection tend to be younger, and alleles with the same / $4 \mathrm{Ns}$ / value but different $4 \mathrm{Ns}$ value differ in the mean and standard deviation of their allele ages (Figure 4C). The distributions of pairwise coalescent times for allele carriers show concordant patterns (Figure 4D): alleles under the stronger positive selection had, on average, younger $T_{2}$ values than negatively selected alleles of the same magnitude. Further, when we contrasted the $T_{2}$ distribution of the negatively selected alleles inspected $(4 N s=-50,-100)$, we saw that their mean $T_{2}$ value did not differ much, and their biggest difference was due to a slightly smaller standard deviation in the most deleterious allele (Figure 4D).

We next used our method to infer the strength of selection for this expansion scenario and found that it can provide approximately unbiased estimates of the sign and strength of selection (Figure 5, using 10,000 realized values of $L$ from 10,000 pairs of haplotypes at independent loci). This does not mean we can differentiate between positive and negative selection in all non-equilibrium models. The power to do so will be dependent on the parameters of the non-equilibrium demography being studied. As an example, in an ancient bottleneck scenario we find there are no significant differences in the distribution of $T_{2}$ between alleles that have the same absolute strength of selection, indicating that we would not be able to differentiate between alleles under positive or negative selection under this demographic model

305 (Supplementary Figure S1).
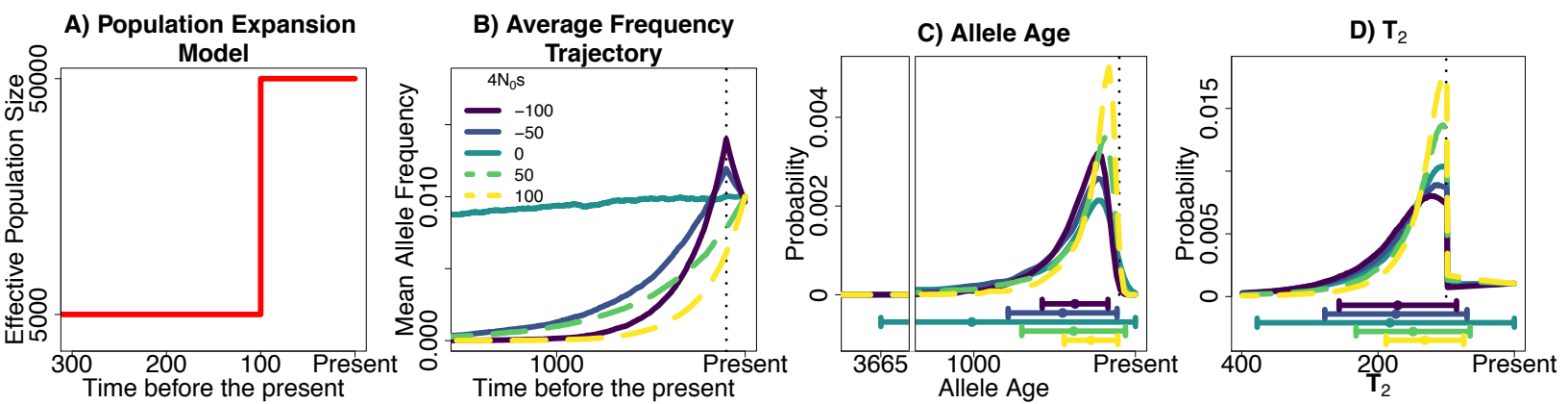

Figure 4.- Properties of alleles sampled at a $1 \%$ frequency under different strengths of selection in a population expansion scenario. A) Population expansion model analyzed. B) Mean allele frequency at different times in the past, in units of generations. Note that alleles under the same absolute strength of selection ( $4 N s$ ) have very different average allele frequency trajectories, in contrast to the constant population size scenario (Fig 2); C) Probability distribution of allele ages and D) Probability distribution of pairwise coalescent times $T_{2}$. The dot 
and whiskers below $C$ ) and $D$ ) represent the mean value of the distribution and the two whiskers extend at both sides of the mean until max(mean +- s.d. ,0).

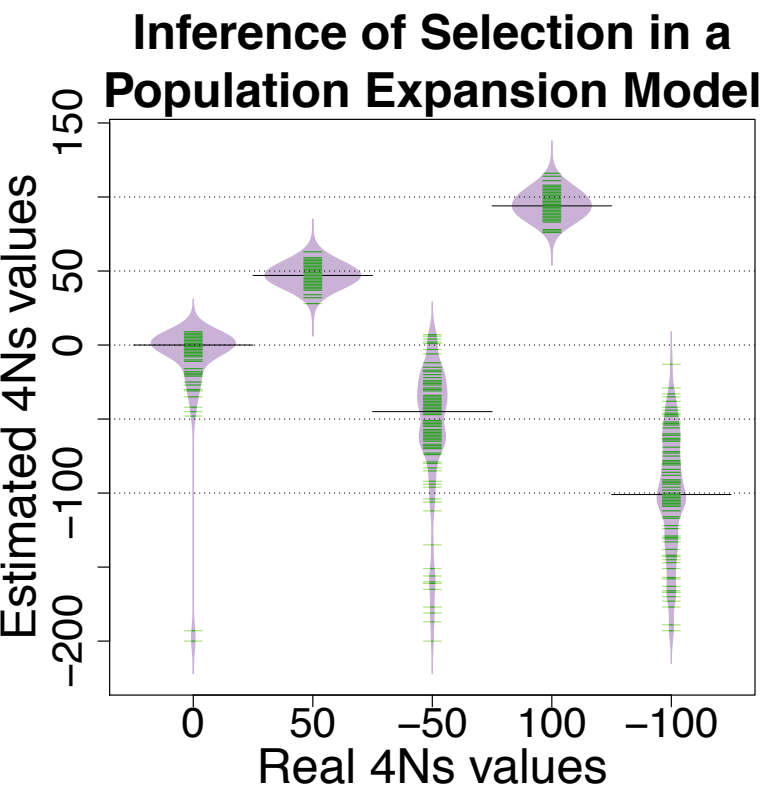

Figure 5.- Estimation of the strength of natural selection in a population expansion model for $1 \%$ frequency alleles. The green lines indicate one estimated value of $4 N s$. 'Real $4 N s$ values' indicate the $4 N s$ values used in the simulations and 'Estimated $4 N s$ values' refers to the values estimated by our method. The median value of the estimates of $4 \mathrm{Ns}$ is shown with a solid line. The recombination rate in the simulated $250 \mathrm{~kb}$ region for the most recent epoch was set equal to $\rho=4 N r=1,000$ and the mutation rate was set equal to $\theta=4 N u=1,000$.

\section{A method for inference of the distribution of fitness effects for variants found at a} particular frequency (" $D F E_{f}$ ")

Our composite likelihood framework is extendible to find the distribution of fitness effects $D F E_{f}$ for a set of variants at a particular frequency $f$. This distribution, which we denote as $D F E_{f}$, is 332 mutations that recently entered the population. To parameterize the $D F E_{f}$ we use a discretized, 333 partially collapsed gamma distribution following studies that use a gamma distribution (Boyko et 334 al. 2008; Kim et al. 2017). We parameterize the gamma component with two parameters that 335 represent the shape $\alpha$ and scale $\beta$. We discretize the distribution to cover only integer values of $3364 N s$ for computational reasons, and then collapse the probabilities for all values greater than a 337 threshold $4 N s$ value (which we denote as $\tau$ ) to a single point mass. The point mass probability is necessary to facilitate the integration over $4 N s$ values when computing $\mathcal{L}\left(\alpha, \beta, D, f \mid L \in w_{i}\right)$. We 
denote the resulting distribution as $D F E_{f}(\alpha, \beta)$. In practice, we explore different values of $\alpha$ and

$340 \quad \beta$ while keeping the value of $\tau$ fixed to a large value (i.e 300), effectively representing strongly

341 selected variants (see Methods).

342 The likelihood of having a certain distribution of identity by state lengths $L$ given a 343 demographic scenario $D$, a variant at a frequency $f$ and two parameters $\alpha$ and $\beta$ is equal to:

$$
\mathcal{L}\left(\alpha, \beta, D, f \mid L \in w_{i}\right)=\int_{4 N s=0}^{\tau} P\left(L \in w_{i} \mid 4 N s, f, D\right) P(4 N s \mid \alpha, \beta) d 4 N s
$$

Where $P\left(L \in w_{i} \mid 4 N s, f, D\right)=\mathcal{L}\left(4 N s, f, D \mid L \in w_{i}\right)$ and was introduced in equation 1.

Testing the inference of the distribution of fitness effects for variants found at a particular frequency (" $D F E_{f}$ ")

350 We tested if the distribution of haplotype lengths $L$ can be used to estimate the parameters that define the distribution of fitness effects of variants at a particular frequency. We used distributions of $100,000 \mathrm{~L}$ values obtained via simulations under the constant population size and population expansion demographic model from the past sections under two distributions of

354 fitness effect of new mutations estimated in different species: one from humans (shape $=0.184$; scale $=319.8626 ; \mathrm{N}=1000$ ) (Boyko et al. 2008) and another one from mice (shape = 0.11; scale $=8636364 ; N=1000000$ ) (Halligan et al. 2013).

We found that the estimated parameters of the shape $(\alpha)$ and scale $(\beta)$ of the $D F E_{f}$ of

$3581 \%$ frequency variants in a sample of 4,000 chromosomes have considerable variation (Figure $3596 \mathrm{~A}, \mathrm{~B})$. However, the estimated shape and scale of the $D F E_{f}$ typically imply the correct mean 360 value of the $D F E_{f}$ (estimates lie along the red-dashed lines in Figure 6). This can be better seen 361 in Supplementary Figure $\mathrm{S} 2$. We found that the estimated $D F E_{f}$ parameters on constant 362 population sizes define a $D F E_{f}$ with a mean $4 N s$ value that, on average, is almost equal to the 363 mean $4 N s$ value found across 50,000 simulated $1 \%$ frequency variants. In a population 364 expansion scenario (Figure 6C,D), the estimated $D F E_{f}$ parameters imply a $D F E_{f}$ with a mean $3654 N s$ value that is slightly lower than the actual mean $4 N s$ value, and with considerably higher 366 variance in the estimated mean (Supplementary Figure S2). 

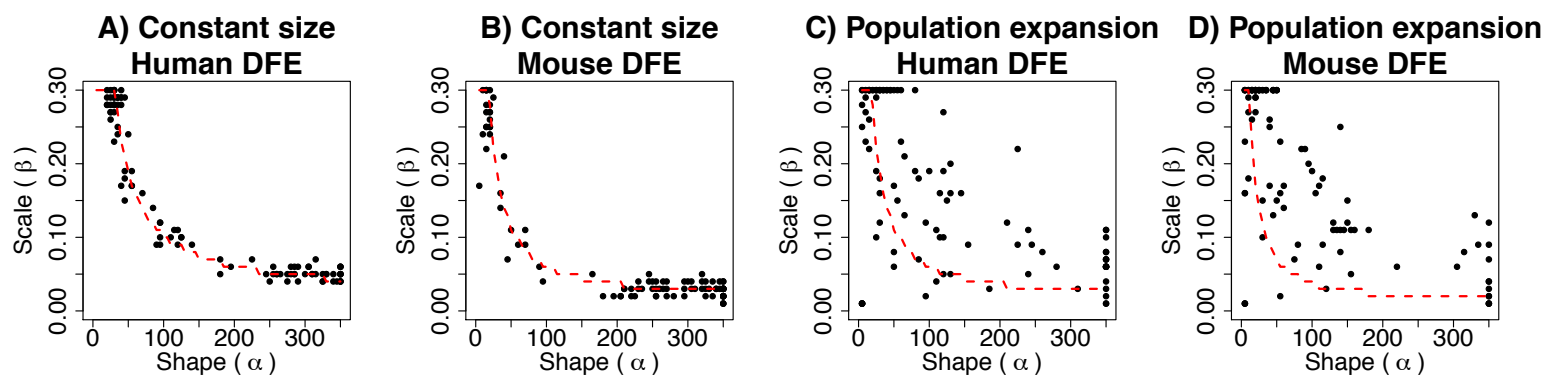

Figure 6.- MLEs of the parameters that define the distribution of fitness effect for variants at a $1 \%$ frequency. We tested if our method was capable of estimating the parameters of the $D F E_{f}$ of variants at a particular frequency in two demographic models and two DFE's. The shape $(\alpha)$ and scale $(\beta)$ parameters define the compound $D F E_{f}$ distribution. Each black dot represents the $\alpha$ and $\beta$ parameter estimated using a set of $100,000 \mathrm{~L}$ values simulated independently. The dotted red line represents a combination of shape and scale parameters from a gamma distribution that give an identical mean $4 N s$ value to the mean $4 N s$ value of the underlying $D F E_{f}$. The grid of scale parameters explored goes from $(0.01,0.02, \ldots, 0.3)$ and the grid of shape parameters explored goes from $(5,10, \ldots, 350)$.

Method for inferring the distribution of fitness effects of new mutations (DFE) from the distribution of fitness effects for variants at a particular frequency $\left(D F E_{f}\right)$

The distribution of fitness effects of variants at a particular frequency $\left(D F E_{f}\right)$ is related to the distribution of fitness effects of new variants $D F E$ by equation 4 (see Methods for more detail):

$$
P_{\psi}\left(\boldsymbol{s}_{j} \mid D\right)=P_{\psi}\left(\boldsymbol{s}_{j}\right)=\frac{P_{\psi}\left(\boldsymbol{s}_{j} \mid f, D\right) P_{\psi}(f \mid D)}{P_{\psi}\left(f \mid \boldsymbol{s}_{j}, D\right)}
$$

where $s_{j}$ is an interval of $4 N s$ values $\left[4 N s_{0}, 4 N s_{1}\right) . s_{0}$ and $s_{1}$ define two different selection coefficients. We used a set of non-overlapping intervals $s=\left\{\left[4 N s_{0}, 4 N s_{1}\right),\left[4 N s_{1}, 4 N s_{2}\right),\left[4 N s_{2}\right.\right.$, $\left.\left.4 N s_{3}\right) \ldots,\left[4 N s_{b-1}, 4 N s_{b}\right)\right\}=\left\{\boldsymbol{s}_{1}, \boldsymbol{s}_{2}, \boldsymbol{s}_{3}, \ldots, \boldsymbol{s}_{\boldsymbol{b}}\right\} . \psi$ is a vector of the parameters $\psi=\left\{\psi_{1}, \psi_{2}, \psi_{3}, \ldots, \psi_{\kappa}\right\}$ that define the $D F E$.

The probabilities $P_{\psi}\left(\boldsymbol{s}_{\boldsymbol{j}} \mid f, D\right)$ over all the intervals in $s$ define the distribution of fitness effects of variants at a particular frequency $D F E_{f}$ over a set of discrete bins. After inferring the $D F E_{f}$ using our composite likelihood method, we can calculate $P_{\psi}\left(\boldsymbol{s}_{\boldsymbol{j}} \mid f, D\right)$ from the inferred $D F E_{f}$. On the other hand, $P_{\psi}\left(\boldsymbol{s}_{j} \mid D\right)=P_{\psi}\left(\boldsymbol{s}_{\boldsymbol{j}}\right)$ since the demographic scenario $D$ does not change the proportion of new variants in a selection interval $s_{j} \cdot P_{\psi}\left(s_{j}\right)$ defines the proportion of new mutations inside a $\boldsymbol{s}_{\boldsymbol{j}}$ interval. It is equal to the $D F E$ over a set of discrete intervals $\boldsymbol{s}_{\boldsymbol{j}}$. Regarding the other two probabilities shown in the equation, $P_{\psi}(f \mid D)$ can be estimated by 
measuring the proportion of variants at a certain frequency $f$ given $D$ and a set of parameters $\psi$ that define the DFE. $P_{\psi}\left(f \mid \boldsymbol{s}_{j}, D\right)$ can be computed via simulations (see Supplementary Text for more details).

Testing inference of the distribution of fitness effects of new mutations $D F E$ from the

We estimated the distribution of fitness effects of new mutations, i.e. the $D F E$, in a population expansion scenario given the distribution of fitness effects $D F E_{f}$ of a set of variants at a $1 \%$ frequency (Figure 7 - Boyko Human DFE; and Supplementary Figure S3 - Human DFE with a scale value that is 20 times smaller). We see that the inferred and real $P_{\psi}\left(\boldsymbol{s}_{\boldsymbol{j}}\right)$ values match using equation (4), with some slight discrepancies that could be due to either using a $\boldsymbol{s}_{\boldsymbol{j}}$ bin that 410 is not small enough or small inaccuracies in the estimated probabilities of $P_{\psi}\left(\boldsymbol{s}_{\boldsymbol{j}} \mid f, D\right), P_{\psi}(f \mid D)$ 411 or $P_{\psi}\left(f \mid s_{j}, D\right)$. We also note that variants at a $1 \%$ frequency tend to be less deleterious 412 compared to new variants based on the comparison of the distributions $P_{\psi}\left(\boldsymbol{s}_{j} \mid f, D\right)$ against $413 P_{\psi}\left(s_{j}\right)$. Additionally, we used our $D F E_{f}$ estimates from Figure 6 to estimate $P_{\psi}\left(s_{j}\right)$. The $P_{\psi}\left(s_{j}\right)$ 414 estimates are accurate, but display a larger variance under the population expansion scenario compared to the constant size scenario (Supplementary Figure S4).

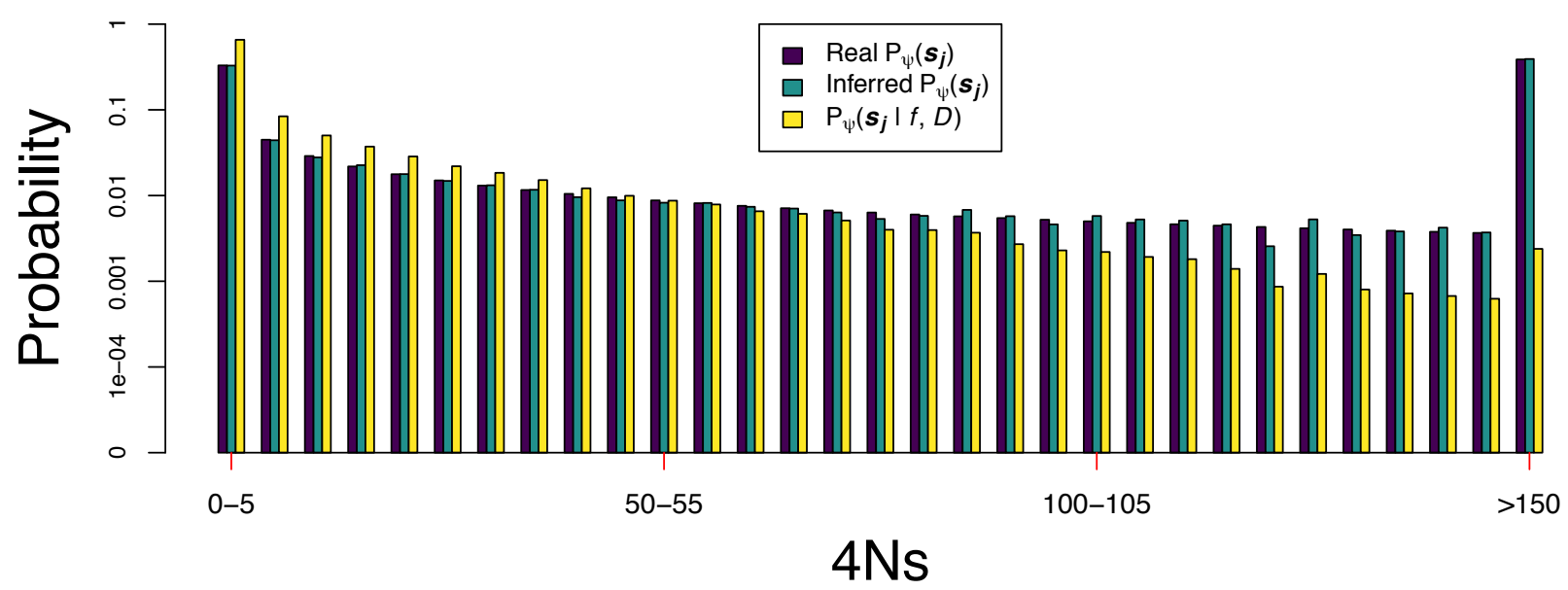

Figure 7.- Inference of the distribution of fitness effects of new mutations from the distribution of fitness effects of variants at a certain frequency in deleterious variants. The DFE follows a gamma distribution with shape and scale parameters equal to 0.184 and 1599.313, respectively. This is equal to the gamma distribution inferred by Boyko et al. (2008) 
demographic model has a population that grows from 5,000 to 50,000 individuals in the last 100 generations (see also Figure 4A). 'Real $\boldsymbol{P}_{\boldsymbol{\psi}}\left(\boldsymbol{s}_{\boldsymbol{j}}\right)$ ' refers to the probability of having a $4 N s$ value in a certain interval $\boldsymbol{s}_{\boldsymbol{j}}$ given the distribution of fitness effects of new mutations with parameters $\boldsymbol{\psi}$. ' $\boldsymbol{P}_{\boldsymbol{\psi}}\left(\boldsymbol{s}_{\boldsymbol{j}} \mid \boldsymbol{f}, \boldsymbol{D}\right)$ ' is the probability of having a $4 N s$ value in an interval $\boldsymbol{s}_{\boldsymbol{j}}$ given the distribution of fitness effects $D F E$ with parameters $\psi$ and the demographic scenario $D$ in $f=1 \%$ frequency variants. We calculated $\boldsymbol{P}_{\boldsymbol{\psi}}\left(\boldsymbol{s}_{\boldsymbol{j}} \mid \boldsymbol{f}, \boldsymbol{D}\right)$ from a set of $\sim 40,0004 N s 1 \%$ variants obtained via $P R e F e r S i m$ simulations under the DFE and the population expansion scenario (see Supplementary Text). 'Inferred $\boldsymbol{P}_{\boldsymbol{\psi}}\left(\boldsymbol{s}_{\boldsymbol{j}}\right)$ ' is an estimate of the probability of having a $4 N s$ value in a certain interval $s_{j}$ given the distribution of fitness effects of new mutations with parameters $\psi$ using $\boldsymbol{P}_{\psi}\left(\boldsymbol{s}_{j} \mid \boldsymbol{f}, \boldsymbol{D}\right)$ and equation 4 . The selection coefficient $s$ refers exclusively to the action of deleterious variants in this plot.

Application: Inference of the distribution of fitness effects of $1 \%$ frequency variants in the UK10K dataset

We inferred the distribution of fitness effects of the $2731 \% \pm 0.05 \%$ frequency variants at nonCpG nonsynonymous sites that are more than $5 \mathrm{Mb}$ away from the centromere or telomeres in the phased UK10K haplotype reference panel. The panel was statistically phased with Shapeit2

442 (Delaneau et al. 2013b), which previous analyses have shown produces a low haplotype 443 phasing error (switch error rate approximately < 2.0\%) for low-frequency alleles (Delaneau et al. 444 2013a). Our method assumes that phasing errors will be similar in the nonsynonymous and 445 synonymous variants, implying that differences in the distribution of $L$ will be due to selection 446 instead of phasing errors. We discarded a set of related individuals along with other individuals 447 with no clear European ancestry from the haplotype panel, as previously defined (Walter et al. 448 2015). In the end, we obtained a set of 3,621 individuals (7,242 haplotypes) from the UK10K 449 haplotype panel.

We used an $A B C$ algorithm to infer the demographic scenario that explains the 451 distribution of $L$ for the 152 non-CpG synonymous variants at a $1 \% \pm 0.05 \%$ frequency that are 452 more than $5 \mathrm{Mb}$ away from the centromere or telomeres (see Supplementary Methods, 453 Supplementary Figure S5). CpG sites were removed before estimating $L$ around the non-CpG 454 synonymous sites. We removed CpG sites by excluding sites preceded by a $\mathrm{C}$ or followed by a $455 \mathrm{G}$ (McVicker et al. 2009). Due to computational reasons, in the $A B C$ method we scaled the 456 population size down by a factor of five while increasing the mutation rate $\mu$, selection coefficient $457 s$ and recombination rate $r$ by the same factor of five to keep $4 N s, \Theta=4 N \mu$ and $\rho=4 N r$ 458 constant. That same scaling was used in all the simulations described in this section and in our 459 inference of selection in the UK10K data. We will refer to the inferred scaled model as the 
460 'scaled UK10K model' and we will refer to the model without the scaling as the 'UK10K model'.

461 We find that in the upstream and downstream $250 \mathrm{~kb}$ regions surrounding the 152 synonymous

$4621 \%$ frequency variants and the 273 nonsynonymous $1 \%$ frequency sites there is a similar 463 proportion of exonic sites (Mann-Whitney $U$ test $p$-value $=0.876$ ), PhastCons element sites

464 (Mann-Whitney $U$ test $p$-value $=0.299$ ), and the average strength of background selection

465 (Mann-Whitney $U$ test $p$-value $=0.605$ ) based on the $B$ values (McVicker et al. 2009). The

466 distributions of $B$ values indicate that similar strengths of background selection are acting on the

467 synonymous and nonsynonymous sites, and should reduce genetic variation similarly on

468 regions surrounding both categories of sites. Therefore, the demographic model we inferred for

469 the synonymous variants can be used to model the evolution of the nonsynonymous variants

470 since the reduction in genetic variation due to background selection is similar on the haplotypes

471 surrounding both types of variants (Supplementary Figure S6). The approach of inferring the

472 demographic model using synonymous sites is not novel for analyses with the site frequency

473 spectrum and helps control for the effects of background selection (Boyko et al. 2008; Huber et

474 al. 2017; Kim et al. 2017; Tataru et al. 2017).

475 We performed simulations under the scaled UK10K model inferred using the $A B C$ 476 algorithm. We found that the frequency trajectories and allele ages are significantly different 477 between alleles under different strengths of selection (Figure 8). However, the distribution of $T_{2}$ 478 values is very similar for deleterious alleles that experience up to a twofold difference in the 479 amount of selection acting upon them. This is important to note since the distribution of $T_{2}$ 480 values is one of the most important factors, along with the mutation and recombination rate, 481 determining the resolution of our approach to infer selection.

482 We also performed simulations to analyze if the amount of information present in the 483 UK10K dataset was sufficient to infer selection coefficients in $1 \%$ frequency variants. Our 484 approach takes into account the differences in recombination rates on the regions surrounding 485 each variant on the genome in the UK10K data (Supplementary Methods). We performed 100 486 simulation replicates, where each replicate mimics the amount of information present in the 487 UK10K dataset. Each replicate contains 273 independent loci with 72 haplotypes containing the 488 derived allele. The recombination rates, both to the left and right side of the loci, were assigned 489 based on the average per base recombination rate in the $250 \mathrm{~kb}$ region surrounding each 490 variant (see Supplementary Figure S7). We calculated $L$ moving upstream and downstream of 491 the focal loci, obtaining $\left(\begin{array}{c}72 \\ 2\end{array}\right) \times 2 \times 273 L$ values for each simulation replicate. Using data 492 simulated under 5 different selection coefficients, we found that we were able to obtain accurate 
estimates of selection when the variants were neutral or under positive selection. When we simulated deleterious variants, we found that our estimates of selection tended to be biased towards being more neutral than the actual $4 N s$ value. However, the true value was within the $10^{\text {th }}$ and $90^{\text {th }}$ percentile of the distribution of estimated values (Supplementary Figure S8). We obtained similar results when the simulated 273 loci shared the same recombination rate 498 (Supplementary Figure S9). We obtained equally accurate estimates of $P_{\psi}\left(\boldsymbol{s}_{\boldsymbol{j}}\right)$ on the $\boldsymbol{s}_{\boldsymbol{j}}$ 499 intervals when we performed simulations using the Boyko distribution of fitness effects under 500 the scaled and UK10K demographic model (Supplementary Figure S10-S11; Supplementary 501 Table S2-S3).

502

503
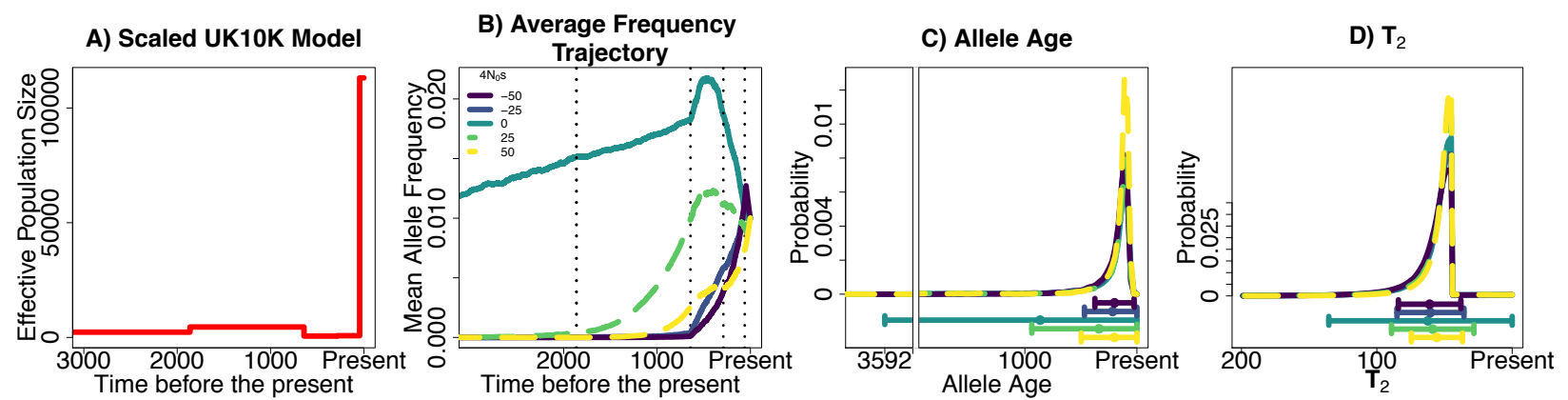

Figure 8.- Properties of alleles sampled at a $1 \%$ frequency under different strengths of natural selection in the scaled UK10K model inferred in the UK10K data. A) Population model inferred in the UK10K dataset. B) Mean allele frequency at different times in the past, in units of generations. C) Probability distribution of allele ages and D) Probability distribution of pairwise coalescent times $T_{2}$. The dot and whiskers below $\mathrm{C}$ ) and $\mathrm{D}$ ) represent the mean value of the distribution and the two whiskers extend at both sides of the mean until max(mean +- s.d. ,0).

We performed bootstrap replicates of the $L$ values from the $2731 \%$ frequency

515 to evaluate the variation in our estimates of $4 N \mathrm{~s}$. We removed $\mathrm{CpG}$ sites before estimating the $L$

516 values surrounding the nonsynonymous and synonymous variants. The variation around the 517 estimates using bootstrap replicates is shown in Supplementary Figure S12, where we see that

518 the point estimates in the replicates tend to be close to a $4 N s$ value equal to 0 for both 519 nonsynonymous and synonymous variants. We performed the inference on the $1 \%$ frequency 520 synonymous variants because an inferred $4 N s$ value that was nominally different from 0 would 521 indicate problems with our methodology such as a misspecified demographic model. 
We used the $L$ values for the 273 nonsynonymous variants at a $1 \%$ frequency to infer

523 the parameters of the distribution of fitness effects $D F E_{f}$. We assume that no derived variants

524 we observe are under positive selection and that the $D F E_{f}$ follows a gamma distribution with a

525 point mass, as explained in the section Inference of the distribution of fitness effects of variants

526 at a particular frequency. When we solved the integral from Equation 3, we used discretized

527 values of $4 N s$ that went from 0 to 75 , and we defined that $P(4 N s=K=-75 \mid \alpha, \beta)=1-$

$528 \sum_{i=0}^{i=-74} P(4 N s=i \mid \alpha, \beta)$. We only explored $4 N s$ values from 0 to -75 because we only had high

529 resolution for those $4 N s$ values (as indicated by ESS values bigger than 100, see

530 Supplementary methods for an explanation of ESS values; Supplementary Figure S13). We

531 inferred a scale value of 0.01 and a shape value of 0.03 . Based on a set of bootstrap replicates,

532 we found that our estimates clustered on the edges of the shape parameter values explored

533 (Supplementary Figure S14). This effect is specific to the inferred demographic scenario for the

534 UK10K dataset, since we did not observe the same phenomenon in the simulations done under

535 the constant population size and population expansion demographic scenarios we explored

536 previously (Figure 6). Based on our estimates of the $D F E_{f}$, we estimated $P_{\psi}\left(\boldsymbol{s}_{\boldsymbol{j}}\right)$ by employing

537 Equation 4 and using $P_{\psi}(f \mid D)$ (see Supplementary Methods for an explanation of our

538 calculation of $P_{\psi}(f \mid D)$ ). We compared those values with previously obtained estimates (Boyko

539 et al. 2008; Kim et al. 2017). The point estimates of $P_{\psi}\left(\boldsymbol{s}_{j}\right)$ along with the $90 \%$ bootstrap

540 percentile intervals for other $\boldsymbol{s}_{\boldsymbol{j}}$ intervals are shown in Figure 9 and Supplementary Figure S15.

541 We also show information for other bootstrap percentile intervals on Supplementary Table S4.

542 Based on our $90 \%$ bootstrap percentile intervals we find that our estimate of $P_{\psi}\left(s_{j} \in[5,50)\right)$ is

543 smaller than the probabilities computed by Boyko et al. 2008 and Kim et al. 2017. On the other

544 hand, the estimate of $P_{\psi}\left(s_{j} \in[50, \infty)\right)$ was bigger than the estimates of Boyko et al. 2008 and

$545 \mathrm{Kim}$ et al. 2017. The probabilities of having a value of selection $s$ over different orders of

546 magnitude are shown on Supplementary Table S5 and are compared with the probabilities

547 obtained by (Boyko et al. 2008; Kim et al. 2017). We also computed p-values under the null

548 hypothesis that there is no difference between the estimated $P_{\psi}\left(\boldsymbol{s}_{j}\right)$ values from the data and

549 the $P_{\psi}\left(s_{j}\right)$ from the Boyko distribution of fitness effects (see Supplementary Figure S16). The

$550 \mathrm{p}$-values were bigger than 0.05 for the three intervals $s_{j} \in[0,5), s_{j} \in[5,50)$ and $s_{j} \in[50, \infty)$.

551 Therefore, the distribution of fitness effects is not different from the distribution of fitness effects

552 estimated by Boyko et al. (2008) over the three $\boldsymbol{s}_{\boldsymbol{j}}$ intervals inspected. 
554

555

556

557

558

559

560

561

562

563

564

565

566

567

568

569

570

571

572

573

574

575

576

577

578

579

580

581

582

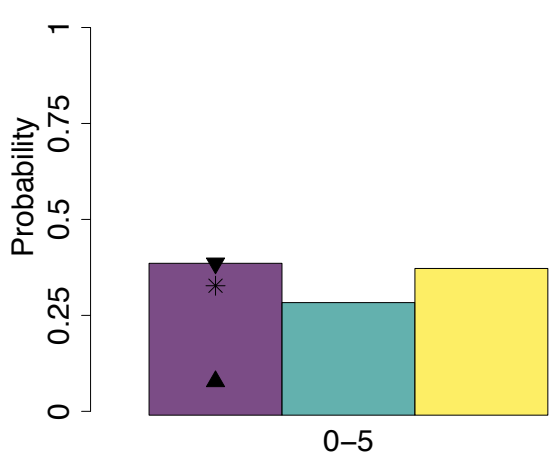

Figure 9.- Inferred distribution of fitness effects of new mutations and $1 \%$ frequency deleterious variants in the UK10K dataset. 'Inferred $P_{\psi}\left(s_{j}\right)$ ' refers to the probability of having a $4 N s$ value in a particular interval $s_{j}$ given the distribution of fitness effects of new mutations DFE. We estimated $P_{\psi}\left(\boldsymbol{s}_{j}\right)$ for the $\boldsymbol{s}_{j}$ interval $=[5,50)$ by summing up the $P_{\boldsymbol{\psi}}\left(\boldsymbol{s}_{\boldsymbol{j}}\right)$ probabilities over the invervals $[5,10),[10,15),[15,20),[20,25),[25,30),[30,35),[35,40),[40,45)$ and $[45$, $50)$. The selection coefficient $s$ refers exclusively to the action of deleterious variants in this plot. We compared our inferences with those of Boyko et al. (2008) and Kim et al. (2017). The two triangles shown in each $\boldsymbol{s}_{j}$ interval denote the upper and lower limit of the $90 \%$ bootstrap percentile interval across 100 bootstrap replicates. The asterisk signs are the mean values for the inferred probabilities $P_{\psi}\left(s_{j}\right)$ calculated from 100 bootstrap replicates. Despite the fact that the estimated Boyko et al $2008 P_{\psi}\left(s_{j}\right)$ values fall outside of the $90 \%$ bootstrap percentile from the inferred $P_{\psi}\left(s_{j}\right)$ in the intervals $s_{j} \in[5,50)$ and $s_{j} \in[50, \infty)$, these differences are not significant according to $p$-values computed under the null hypothesis that there is no difference between the estimated $P_{\psi}\left(\boldsymbol{s}_{\boldsymbol{j}}\right)$ values and the $P_{\psi}\left(\boldsymbol{s}_{\boldsymbol{j}}\right)$ from the Boyko distribution of fitness effects (see Supplementary Figure S16).

\section{Discussion}

We have developed a composite likelihood method to estimate the strength of natural selection acting on alleles at a certain frequency in the population. Our method builds upon previous work showing signatures of higher linkage disequilibrium for putatively deleterious alleles in comparison with neutral alleles (Kiezun et al. 2013). This result was shown to be in line with Takeo Maruyama's work showing that deleterious alleles at a certain frequency tended to be younger than neutral alleles in constant population sizes (Maruyama 1974). Here we introduce a method to estimate the strength of natural selection based on linkage disequilibrium using the pairwise identity by state lengths $L$.

We found that the distribution of $L$ captures differences in the absolute strength of the selection coefficient $4 N s$ in a constant population size scenario. The mean allele frequency trajectory is practically identical for deleterious and advantageous alleles experiencing the same 
583 amount of selection; therefore, any statistic based on haplotype signatures will be insufficient in

584 that scenario to distinguish between positive and negative selection.

585 On the other hand, we found that the distribution of $L$ is sufficient to differentiate between

586 advantageous and deleterious alleles under some non-equilibrium demographic scenarios,

587 including the demographic scenario inferred from the UK10K dataset. This is encouraging, since

588 most natural populations are very likely to have evolved under a non-equilibrium demographic

589 scenario and it is precisely in such scenarios where we would like to be able to differentiate

590 between alleles with different types of selection.

$591 \quad$ The mean allele frequency trajectories of deleterious alleles segregating at a $1 \%$

592 frequency when the population is expanding are particularly noteworthy. These alleles tend to

593 have increased in frequency when the population size is low. Then, they decrease in frequency

594 when the population expands due to a higher efficacy of selection. This suggest that it is likely

595 that, on average, deleterious alleles would tend to come from higher frequencies in the recent

596 past in expanding populations. These simulations of allele frequency trajectories under several

597 demographic scenarios are useful to understand past fluctuations in frequency and haplotypic

598 patterns one might expect for selected alleles. Recent work has analyzed how different

599 summaries of genetic variation change over time in non-equilibrium scenarios (Peischl et al.

600 2013; Lohmueller 2014a; Simons et al. 2014; Do et al. 2015; Henn et al. 2015; Balick et al.

601 2015; Brandvain \& Wright 2016; Marsden et al. 2016; Koch \& Novembre 2017), and analyzing

602 the behavior of frequency trajectories is helpful to understand those changes.

603 When we estimated parameters that define the $D F E_{f}$ of segregating variants, we found

604 that our method can provide reasonable estimates of the parameters that would lead to

605 estimating a sensible value of the mean of the $D F E_{f}$ in several scenarios. Under a constant

606 population size, the scale estimates of the $D F E_{f}$ are inversely correlated with the shape

607 parameters. Note that this curve decay causes the product of the scale and shape parameters

608 to have relatively similar values. Under a population expansion model, the estimates of the

609 shape and scale show a wider variation around the curve than the constant population size

610 scenario (Figure 6). Similarly, the pairwise coalescent time $T_{2}$ distribution between variants with

611 different negative selection coefficients appear more similar to each other in a population

612 expansion scenario as compared to a constant population size scenario (Figure 4D and 2C).

613 Due to the greater variation in the estimates of the parameters that define the $D F E_{f}$ of variants

614 at a $1 \%$ frequency, we also see a larger variation in the mean $4 N s$ values estimated in a

615 population expansion as compared to a constant population size demographic scenario

616 (Supplementary Figure S2). Estimates of the mean $4 N s$ values are more precise under a 
617 constant population size compared to the population expansion scenario. For the UK10K

618 demographic scenario and the scaled UK10K model, where there is a large recent population

619 expansion, we saw that the proportion of $4 N s$ values smaller than 5 tended to be overestimated

620 while the proportion of $4 N s$ values larger than 5 were underestimated based on the analysis of

621 simulations using the Boyko et al. (2008) DFE. The consequence is that the mean $4 N s$ value

622 would tend to be underestimated under the UK10K demographic scenario and the scaled

623 UK10K demographic scenario (Supplementary Figure S10-S11). It is likely that this

624 underestimation will be seen in other scenarios with large recent population expansions.

625 One technical aspect from our methodology that could be subject to future improvement

626 is that the space of scale and shape parameters we explore is limited due to low effective

627 sample size (ESS) values. In the case of the UK10K dataset, the ESS are smaller than 100

628 in $4 N s$ values smaller than -75 (Supplementary Figure S12). To increase the values of the ESS,

629 one possible improvement of our method is to make better proposals for the allele frequency

630 trajectories going backwards in time. That is, to improve our choice of the importance sampling

631 distribution. Future work will be devoted to make improvements in this issue, particularly in

632 populations undergoing recent large expansions. One possibility is to expand the theory of

633 Wright-Fisher bridges to select trajectories that end at a certain frequency $f$ in the present under

634 non-equilibrium scenarios (Schraiber et al. 2013). We did not find the same pattern of low ESS

635 values in the other two demographic scenarios we analyzed, where the population sizes did not

636 experience changes in population size of the same magnitude as in the demographic model

637 inferred in the UK10K data.

638 Using the UK10K data, we obtained a point estimate, along with $90 \%$ bootstrap interval

639 calculations, of the DFE. Our point estimates are consistent with point estimates obtained using

640 information from the site frequency spectrum (Boyko et al. 2008) (Supplementary Figure S16). It

641 is possible that we find discrepancies between the estimated DFE in other species or

642 populations using haplotypic information compared to using data from the site frequency

643 spectrum. In a similar vein, important discrepancies on the inferred past demographic histories

644 on human populations have been found when using site frequency spectrum data and

645 haplotypic information, and some of the potential causes of the differences have been carefully

646 discussed previously (Harris \& Nielsen 2013; Hsieh et al. 2016; Beichman et al. 2017).

647 Technical aspects of the data that can impact the demographic inferences when using

648 haplotypic data include: 1) Switch errors during statistical phasing which cause a bias towards

649 more recent split-time estimates (Song et al. 2017), 2) Uncalled heterozygous sites due to low

650 genomic coverage which causes a bias towards lower effective population size estimates 
651 (Nadachowska-Brzyska et al. 2016), 3) Not filtering low coverage, potentially false positive 652 variants, which can produce poor estimates of sudden contractions or expansions 653 (Nadachowska-Brzyska et al. 2016).

654 With respect to the potential impact of switch errors in our inference, the UK10K project 655 does not report switch error rates, but we would expect them to be even lower than those of the 6561000 Genomes Project (estimated to be $0.56 \%$ with a mean of distance of $\sim 1,062 \mathrm{~kb}$ between 657 errors) (Auton et al. 2015), due to the fact that the UK10K has approximately $50 \%$ more 658 samples than the 1000 genomes project, and all the samples come from the same population. 659 We expect to see the impact of phasing errors to be small in our data since we are using 660 window sizes of $500 \mathrm{~kb}$ in our analysis; this window size is smaller than the mean distance 661 between switch errors in the 1000 Genomes Data, and the mean distance between switch 662 errors is likely to be even larger in the UK10K project.

663 Our inferences of the DFE can be impacted due to the low genomic coverage present in 664 the UK10K dataset ( $4 \mathrm{x}$ on average). However, the estimate of the percentage of genotypes 665 correctly called in the UK10K dataset is equal to $99.688 \%$ for common variants with a frequency 666 bigger than $5 \%$, and $99.999 \%$ for singletons (Walter et al. 2015). This indicates that the 667 sequencing strategy carried out in the UK10K dataset should not have a large impact on our 668 estimates of the DFE due to wrongly called genotypes across individuals.

669 Apart from the technical aspects that could be impacting our estimates of the DFE, there 670 are biological phenomena that could be responsible for differences in the DFE estimates we see 671 when we use site frequency spectrum information and haplotypic data. One of those 672 phenomena is linked selection, which reduces the genetic variation in neutral sites next to an 673 allele under either positive or negative selection (Cutter \& Payseur 2013). Linked selection will 674 increase the lengths of the pairwise haplotype lengths in the synonymous sites used to infer the 675 demographic scenario and in the nonsynonymous sites used to infer the distribution of fitness 676 effects. Previous work estimating the distribution of fitness effects using site frequency spectrum 677 information has shown that using synonymous sites to estimate the demographic scenario 678 controls for the effect of linked selection and gives an accurate estimation of the DFE (Huber et 679 al. 2017). We expect the same effect to take place when using haplotypic information. 680 Specifically the amount of linked selection is predicted to be similar between synonymous and 681 nonsynonymous variants at 1\% frequency (see caption Supplementary Figure S6), indicating 682 that the increase in pairwise haplotype lengths should be similar for both synonymous and 683 nonsynonymous sites. 
Another biological phenomenon that could impact our DFE estimates is the incompleteness of the demographic model fitted to the data (Harris \& Nielsen 2013; Garud et al. 686 2015; Beichman et al. 2017). We are fitting a demographic model with one deme to the UK10K 687 dataset, and it is possible that fitting a model with population structure could give a better fit to 688 the haplotypic data and to the site frequency spectrum data (Harris \& Nielsen 2013). We also 689 are not modelling non-crossover gene conversion (Andolfatto \& Nordborg 1998; Korunes \& 690 Noor 2017). Non-crossover gene conversion events involve haplotype tracts of approximately $691100-1000 \mathrm{bp}$ and the probability that any site in the genome is involved in a non-crossover gene 692 conversion event is $5.9 \times 10^{-6} / \mathrm{bp} /$ generation (Williams et al. 2015). Their impact is to break 693 down linkage disequilibrium, which in our model, for a single variant would result in inferences 694 that are biased towards neutrality; however, in aggregate if it impacts LD around synonymous 695 and nonsynonymous variants equally, the effect on inferences may be minor. Nonetheless, 696 modelling noncrossover gene conversion could improve models of the haplotype signatures of 697 selection.

698 As another factor, changes on the DFE over time could lead to differences in the inferred 699 DFE from the site frequency spectrum and the haplotypic data. DFE estimates from the site 700 frequency spectrum data use information from variants that have appeared across a broad 701 range of time. On the other hand, the haplotype data we used comes from 1\% frequency 702 variants that have appeared recently. The relaxation of selective pressures across time is one 703 way to change the selective coefficient of variants to make them more neutral (Somel et al. 704 2013; Lynch 2016). Our results argue in favor of conserved selective coefficients over time in 705 humans, in line with recent results (Fortier et al. 2019).

$706 \quad$ Although here we analyzed the distribution of fitness effects of nonsynonymous variants 707 at a certain frequency, it is possible to determine the distribution of fitness effects of variants 708 within specific functional categories. One possibility is to try to determine the strength of 709 selection of alleles on variants that are predicted to be more deleterious based on the Fitcons 710 (Gulko et al. 2015), SIFT (Sim et al. 2012), Polyphen (Adzhubei et al. 2010) or C-scores

711 (Kircher et al. 2014; Racimo \& Schraiber 2014). It is also be possible to estimate the strength of 712 selection in a set of alleles that have a particular collection of genomic features (Huang \& Siepel 713 2019). This can help us to obtain genome-wide estimates of the selection coefficient of variants 714 based on their predicted functional category. This is of particular interest to genome-wide 715 association studies, due to the interest in understanding the association between associated 716 variants and their selection coefficients on different complex traits. Additionally the use of the 717 newly developed tree-sequence framework (Kelleher et al. 2018; Haller et al. 2019) for 
718 simulations should also help to speed up the calculation of the likelihood of different values of

719 selection in the part of our method that depends on Monte Carlo simulations. Another future

720 avenue of research is to infer the distribution of selection coefficients of new mutations

721 combining information from the $D F E_{f}$ inferred at many different frequencies in the population.

722 Combining information from variants at many frequencies is likely to increase the accuracy of

723 estimates of the distribution of fitness effects of new variants, and could detect changes in the

724 distribution of fitness effects of new variants through time.

725

726 Methods

\section{Inference of selection}

The likelihood of having a particular selection coefficient $4 N s$ conditioning on the allele

731 frequency $f$ and the demographic scenario $D$ using information from one length $L \in w_{i}$ can be

732 estimated as:

$$
\mathcal{L}\left(4 N s, f, D \mid L \in w_{i}\right)=\int P\left(L \in w_{i} \mid H_{i}\right) P\left(H_{i} \mid 4 N s, f, D\right) d H_{i}
$$

where $H_{i}$ is a particular allele frequency history, i.e. a trajectory of allele counts from when the allele first appears in the population until the present. We can compute $P\left(L \in w_{i} \mid H_{i}\right)$ via Monte Carlo simulations done using mssel (Kindly provided by Richard Hudson), which assumes the structured coalescent model to simulate haplotypes containing a site whose frequency trajectory is determined by $H_{i}$. We used mssel to simulate many pairs of haplotypes $(10,000$ independent

740 pairs for all scenarios but the UK10K scenario, where we simulated 273 independent sets of 72 741 haplotypes) given an allele frequency trajectory $H_{i}$ and we computed the $L$ value for each pair of 742 haplotypes. We can use that distribution of $L$ values for a given allele frequency $H_{i}$ to find the 743 probability $P\left(L \in w_{i} \mid H_{i}\right)$ that $L$ falls in a certain window $w_{\mathrm{i}}$. It is important to appreciate that these 744 Monte Carlo simulations can include additional information about the recombination rate present 745 in a particular region. Using the appropriate recombination rate is important because it changes 746 the values of $L$.

$747 \quad$ The likelihood $\mathcal{L}(4 N s, f, D \mid L)$ is found by integrating over the space of allele frequency 748 trajectories that end at a frequency $f$ in the present and have a selection coefficient $4 N s$. One 749 possible way to perform that integration step is to perform many simulations under the 
assumptions of the Poisson Random Field framework (Sawyer \& Hartl 1992; Hartl et al. 1994) $(P R F)$ and utilize rejection sampling to only keep those trajectories that end at a frequency $f$ in

752 the present. Under the PRF model, the number of mutations that enter the population each 753 generation $i$ have a Poisson distribution with mean $2 N_{i} \mu K=\Theta / 2$, where $N_{i}$ is the population

754 size in generation $i, \mu$ is the mutation rate per base and $K$ is the number of sites being 755 simulated. The sites are independent and the frequency of each mutation changes each 756 generation following a Wright-Fisher model with selection. We could generate many allele 757 frequency trajectories under this framework given a particular value of $4 N s$ and just keep those 758 trajectories that end at a frequency of $f$. However, this is inefficient and computationally 759 demanding, since the vast majority of allele frequency trajectories will not end at a frequency $f$ in 760 the present. And it is particularly more challenging if we wish to calculate $\mathcal{L}(4 N s, f, D \mid L)$ for a 761 grid of values of $4 \mathrm{Ns}$. In the next section we show an alternative importance sampling approach 762 we developed to perform an efficient integration over the space of allele frequency trajectories 763 given $4 N s$ and $f$.

\section{Integration over the space of allele frequency trajectories using importance sampling}

We used importance sampling to integrate over the space of allele frequency trajectories and 768 calculate the likelihood $\mathcal{L}(4 N s, f, D \mid L)$ over many different values of $4 N s$. The efficient 769 integration over the space of allele frequency trajectories is done using the importance sampling approach developed by Slatkin (2001) with a modification regarding the importance sampling distribution we use. Here, the "target" distribution $f(x)=P\left(H_{i} \mid s, f\right)$ are samples of allele frequency trajectories that end at a frequency $f$ and have a selection coefficient $s$. number of copies of the allele a present each generation since the allele appeared in the population. Therefore, $H_{i}=\left\{i_{T}, i_{T-1}, i_{T-2}, \ldots, i_{2}, i_{1}, i_{0}\right\}$, where $i_{T}=0$ and $i_{T-1}=1$. The effective population sizes at those times are $N=\left\{N_{T}, N_{T-1}, N_{T-2}, \ldots, N_{2}, N_{1}, N_{0}\right\}$. The allele appears in generation $T$-1, where it has 1 copy in the population.

We define the fitness of the genotypes $A A, A a$ and aa as $1,1+s$ and $1+2 \mathrm{~s}$, respectively.

779 Under a Wright-Fisher model with selection, the probability of moving from $i_{t}$ to $i_{t-1}$ copies of 780 the allele going forward in time is equal to:

781

782

$$
P\left(i_{t-1} \mid i_{t}\right)=p_{i_{t}, i_{t-1}}=\left(\begin{array}{c}
2 N_{t-1} \\
i_{t-1}
\end{array}\right) x_{t}^{\prime i_{t-1}}\left(1-x_{t}^{\prime}\right)^{2 N_{t-1}-i_{t-1}}
$$


783 where

784

$785 \quad x_{t}^{\prime}=x_{t} \frac{1+2 s x_{t}+s\left(1-x_{t}\right)}{1+2 s x_{t}^{2}+2 s x_{t}\left(1-x_{t}\right)}$.

786 The frequency of the allele at generation $t$ is $x_{t}=\frac{i_{t}}{2 N_{t}}$.

787 As a "importance sampling" distribution $g(X)$, we use a very similar process to a Wright-

788 Fisher neutral model. We start with the count $y$ of the number of derived alleles $a$ in the present

789 based on a sample of $n$ alleles. Estimating the frequency in generation 0 based on that sample

790 of alleles is equal to the problem of estimating a probability based on binomial data. Therefore,

791 we can follow Gelman et al. (2013) to state that the posterior density of the distribution of allele

792 frequency $\hat{f}$ in generation 0 is distributed as: $\hat{f} \mid y \sim \operatorname{Beta}(y+1, n-y+1)$. Based on the

793 distribution of $\hat{f}$, we can obtain the distribution of the number of alleles in generation $0, i_{0}$, just

794 by multiplying $i_{0}=\hat{f} n$ and rounding $i_{0}$ to a discrete value. Then we can define the probability of

795 having $i_{0}$ alleles in generation 0 given that we sampled $y$ derived alleles in a sample of $n$ alleles

796 as:

797

798

$P\left(i_{0} \mid n, y\right)=P\left(X<\frac{i_{0}+0.5}{2 N_{0}} \mid \operatorname{Beta}(y+1, n-y+1)\right)-P\left(X<\frac{i_{0}-0.5}{2 N_{0}} \mid \operatorname{Beta}(y+1, n-y+1)\right)$.

799 On the other hand, the probability that we obtain $y$ derived alleles in a sample of $n$ alleles given

800 that the number of derived alleles in the population is $i_{0}$ is:

801

802

$P\left(n, y \mid i_{0}\right)=\left(\begin{array}{l}n \\ y\end{array}\right)\left(\frac{i_{0}}{2 N_{0}}\right)^{y}\left(1-\frac{i_{0}}{2 N_{0}}\right)^{n-y}$

803

804 After we sample from that distribution, we move backwards in time assuming that the allele is 805 neutral. Under this proposal distribution, if $i_{t-1}=1$, then $i_{t}$ can take any value from 0 to $2 N_{t}$. If $806 i_{t-1}=0$ or $2 N_{t}$ then we stop the allele frequency trajectory. If $i_{t-1}$ is bigger than 1 and smaller 807 than $2 N_{t}$, then $i_{t}$ can take any value from 1 to $2 N_{t}$. These three rules are used together to make 808 sure that each trajectory going forward in time always goes from 0 to 1 copy of the allele.

809 Under the importance sampling distribution we use, the transition probabilities of going 810 from $i_{t-1}$ alleles in generation $t-1$ to $i_{t}$ alleles in generation $i_{t}$ is: 


$$
P\left(i_{t} \mid i_{t-1}\right)=q_{i_{t-1}, i_{t}}= \begin{cases}\frac{\left(\begin{array}{c}
2 N_{t} \\
i_{t}
\end{array}\right) x_{t-1}^{i_{t}}\left(1-x_{t-1}\right)^{2 N_{t}-i_{t}}}{1-\left(\begin{array}{c}
2 N_{t} \\
i_{t}
\end{array}\right) x_{t-1}^{0}\left(1-x_{t-1}\right)^{2 N_{t}}} & \text { if } i_{t-1}=\left(2,2 N_{t}\right) \text { and } i_{t}>0 \\
\left(\begin{array}{c}
2 N_{t} \\
i_{t}
\end{array}\right) x_{t-1}^{i_{t}}\left(i-x_{t-1}\right)^{2 N_{t}-i_{t}} & \text { if } i_{t-1}=1 \\
0 & \text { if } \left.1) i_{t-1}=0 \text { or } 2 N_{t} ; 2\right) i_{t-1}=\left(2,2 N_{t}\right) \text { and } i_{t}=0\end{cases}
$$

815 Where $x_{t-1}=\frac{i_{t-1}}{2 N_{t-1}}$. By generating an allele frequency trajectory with this importance sampling 816 distribution, we can calculate the probability of any sample from this importance sampling 817 distribution $g(x)$ :

$$
g(x)=P\left(i_{0} \mid n, y\right) \prod_{t=1}^{T} q_{i_{t-1}, i_{t}}
$$

818 Finally, the probability of the whole allele frequency trajectory $H_{i}$ going forward in time is then 819 equal to:

820

$$
P\left(H_{i} \mid s, f\right)=f(x)=P\left(n, y \mid i_{0}\right) \prod_{t=T-1}^{1} p_{i_{t}, i_{t-1}}
$$

821

822 Now that we have defined how to sample allele frequency trajectories using our proposal 823 distribution, we can compute the weight for every simulated allele frequency trajectory $H_{i}$ from

$824 g(x)$ as $\omega_{i}=\frac{f\left(x_{i}\right)}{g\left(x_{i}\right)}$. For some of the proposed trajectories under $g(x)$, the trajectory will end up at 825 a frequency of 1 going backwards into the past, instead of 0 . The value of $\omega_{i}$ for those 826 trajectories is defined to be equal to 0.

827 The expected value that we wish to obtain with this problem is $\mathcal{L}\left(4 N s, f, D \mid L \in w_{i}\right)$. After 828 generating $M$ replicates using $g(x)$, we can compute that expected value under the importance 829 sampling framework:

830

$$
\mathcal{L}\left(4 N s, f, D \mid L \in w_{i}\right)=\frac{\sum_{i=1}^{M} \omega_{i} P\left(L \in w_{i} \mid H_{i}\right)}{\sum_{i=1}^{M} \omega_{i}}
$$


832 Using this approach, we can estimate $\mathcal{L}\left(4 N s, f, D \mid L \in w_{i}\right)$ for different values of $s$ using the 833 same set of allele frequency trajectories generated from our importance sampling distribution.

834 This alleviates the need to simulate a different set of allele frequency trajectories for each value 835 of the selection coefficient $s$ that we want to evaluate and follows the idea of a driving value 836 (Fearnhead \& Donnelly 2001). The proposal distribution $g(x)$ is not necessarily optimal for every $837 s$ value, but it is possible to verify if the distribution is reasonable based on the effective sample 838 size (ESS) values (see Equation S1; Supplementary Methods). The ESS indicates the sample 839 size used in a Monte-Carlo evaluation of the target distribution $f(x)$ that is equivalent to the 840 importance sampling approach estimate. Plots of the ESS values for the two main demographic 841 scenarios explored are shown in the Supplementary Figures S18-S19. In every demographic 842 scenario explored, we simulated 100,000 allele frequency trajectories to evaluate 401 values of $8434 N s$ in discrete intervals from -200 to 200. The only values that we need to change to evaluate $844 \mathcal{L}\left(4 N s, f, D \mid L \in w_{i}\right)$ are the importance sampling weights $\omega_{i}$, where we will change the value of $845 P\left(H_{i} \mid s, f\right)=f(x)$ depending on the value of the selection coefficient $s$ evaluated.

$846 \quad$ Finally, given a set of values $\boldsymbol{L}=\left\{L_{1} \in w_{i_{1}}, L_{2} \in w_{i_{2}}, L_{3} \in w_{i_{3}}, \ldots L_{n} \in w_{i_{n}}\right\}$, where $i_{j}$ can take 847 any value from 1 to $S$, we can estimate the composite likelihood of having that set of $L$ values 848 as:

$$
\mathcal{L}\left(4 N s, f, D \mid \boldsymbol{L} \in w_{i}\right)=\prod_{j=1}^{n} \mathcal{L}\left(4 N s, f, D \mid L_{j} \in w_{i_{j}}\right)
$$

\section{Forward-in-time simulations to obtain mean allele frequency trajectories}

853 We used PReFerSim (Ortega-Del Vecchyo et al. 2016) to obtain 10,000 allele frequency 854 trajectories of a $1 \%$ frequency allele under the constant-size demography scenario for 5 855 different values of selection $(4 N s=0,-50,-100,50,100)$. To do those simulations, we performed 856 many replicate simulations where the number of new mutations per generation follows a 857 Poisson distribution with a mean equal to $\Theta / 2=1,000$. Those simulations were repeated until 858 we obtained 10,000 alleles frequency trajectories where the present-day frequency $f$ is equal to $8591 \%$ in a sample of 4,000 chromosomes. We did the same procedure to obtain 10,000 allele 860 frequency trajectories of a $1 \%$ frequency allele for 5 different values of selection $(4 N s=0,-50,-$ $861100,50,100)$ in a population expansion and an ancient bottleneck scenario. The value of $\Theta /$ 
8622 for the most ancestral epoch was set to 1,000 in the population expansion and the ancient

863 bottleneck scenario.

864 In the case of the UK10K demographic scenario, we obtained 10,000 allele frequency

865 trajectories of a $1 \%$ frequency allele for 5 values of selection $(4 N s=0,-25,-50,25,50)$. We

866 performed many simulations using a $\Theta / 2$ value equal to 1,000 for the most ancestral epoch

867 until we obtained 10,000 allele frequency trajectories. We sampled 7,242 chromosomes and

868 retained those trajectories where $f=1 \% \pm 0.05 \%$.

Connecting the distribution of fitness effects of variants at a particular frequency $\left(D F E_{f}\right)$

871 with the distribution of fitness effects of new mutations (DFE)

873 The distribution of fitness effects of variants at a particular frequency $D F E_{f}$ in the population is

874 related to the distribution of fitness effects of new mutations $D F E$ defined by a set of $\kappa$ 875 parameters $\boldsymbol{\psi}=\left\{\boldsymbol{\psi}_{1}, \boldsymbol{\psi}_{2}, \boldsymbol{\psi}_{3}, \ldots, \boldsymbol{\psi}_{\boldsymbol{\kappa}}\right\}$ by the following equation:

876

$$
P_{\psi}\left(f \mid \boldsymbol{s}_{\boldsymbol{j}}, D\right)=\frac{P_{\psi}\left(\boldsymbol{s}_{\boldsymbol{j}} \mid f, D\right) P_{\psi}(f \mid D)}{P_{\psi}\left(\boldsymbol{s}_{\boldsymbol{j}} \mid D\right)}
$$

877 Where we can re-arrange the above equation to obtain:

$P_{\psi}\left(\boldsymbol{s}_{\boldsymbol{j}} \mid D\right)=\frac{P_{\psi}\left(\boldsymbol{s}_{\boldsymbol{j}} \mid f, D\right) P_{\psi}(f \mid D)}{P_{\psi}\left(f \mid \boldsymbol{s}_{\boldsymbol{j}}, D\right)}$

879 The events defined in that formula are:

$f$.- The allele has an $x \%$ sample allele frequency.

881

882

883

884 $\boldsymbol{s}_{\boldsymbol{j}}$ - Allele has a selection coefficient $4 N s$ that falls in the interval $\left[4 N s_{j-1}, 4 N s_{j}\right)$, where $s_{j-1}$

$886 P_{\psi}\left(s_{j} \mid D\right)$ defines the distribution of fitness effects of new mutations over a set of discrete bins 887 when using the information contained across all non-overlapping intervals $\sigma=\left\{\left[4 N s_{0}, 4 N s_{1}\right)\right.$, $\left.888\left[4 N s_{1}, 4 N s_{2}\right),\left[4 N s_{2}, 4 N s_{3}\right) \ldots,\left[4 N s_{b-1}, 4 N s_{b}\right)\right\}=\left\{\boldsymbol{s}_{1}, \boldsymbol{s}_{2}, \boldsymbol{s}_{3}, \ldots, \boldsymbol{s}_{\boldsymbol{b}}\right\}$ covering all $4 N s_{\text {values from } 0}$ 889 to infinite. We defined the endpoints of the first $b-1$ intervals to be equal to $5(i-1)$ and $5 i$, where $i$ 890 takes values from 1 to $b-1$, in all the analysis we performed with the exception of 891 Supplementary Table S4. The last interval was set to be equal to $[5 b, \infty)$. Since $P_{\psi}\left(\boldsymbol{s}_{j} \mid D\right)$ is 
892 independent of the demographic scenario $D$, then $P_{\psi}\left(\boldsymbol{s}_{\boldsymbol{j}} \mid D\right)=P_{\psi}\left(\boldsymbol{s}_{\boldsymbol{j}}\right)$ because $D$ does not 893 impact the proportion of new variants in a selection interval $s_{j}$. If we look at the information of all 894 non-overlapping intervals $\sigma, P_{\psi}\left(\boldsymbol{s}_{j} \mid f, D\right)$ defines the distribution of fitness effects of variants at a 895 particular frequency $D F E_{f}$ over a set of discrete bins. As seen in the section Testing inference of 896 the distribution of fitness effects for variants found at a particular frequency ("DFE $E_{f}$ "), we can 897 use the $\boldsymbol{L}$ values to infer $D F E_{f}$.

$898 \quad P_{\psi}(f \mid D)$ can be computed both in data and in simulations by measuring the proportion 899 of variants at a certain frequency. Calculating $P_{\psi}(f \mid D)$ in genomic data requires us to calculate 900 the proportion of variants at a frequency $f$. That proportion must take into account all variants 901 that have emerged during the demographic history $D$, including variants that have become fixed 902 or have been lost. To calculate $P_{\psi}\left(f \mid \boldsymbol{s}_{\boldsymbol{j}}, D\right)$, we can make the assumption that all the mutations 903 in the interval $\boldsymbol{s}_{\boldsymbol{j}}$ have very similar selection coefficients, which is more likely to be true when the 904 interval is not very big. This probability can be found via forward-in-time simulations, where we 905 simulate variants that have a selection coefficient contained in a certain interval $\boldsymbol{s}_{\boldsymbol{j}}$ in a particular 906 demographic scenario $D$. Then, the proportion of variants in that simulation that have a $f$ 907 frequency in the present is equal to $P_{\psi}\left(f \mid \boldsymbol{s}_{\boldsymbol{j}}, D\right)$.

908 We calculate $P_{\psi}\left(s_{j}\right)$ for the first $b$-1 intervals using Equation 4 . Then, for the last interval $909 \boldsymbol{s}_{\boldsymbol{b}}$ we use $P_{\psi}\left(\boldsymbol{s}_{\boldsymbol{b}}\right)=1-\sum_{i}^{b-1} P_{\psi}\left(\boldsymbol{s}_{\boldsymbol{i}}\right)$. If $\sum_{i}^{b-1} P_{\psi}\left(\boldsymbol{s}_{\boldsymbol{i}}\right)>1.0$, we set the probabilities $P_{\psi}\left(\boldsymbol{s}_{\boldsymbol{j}}\right)=$ $910 P_{\psi}\left(\boldsymbol{s}_{\boldsymbol{j}}\right) / \sum_{i}^{b-1} P_{\psi}\left(\boldsymbol{s}_{\boldsymbol{i}}\right)$ for the first $b$-1 intervals and $P_{\psi}\left(\boldsymbol{s}_{\boldsymbol{j}}\right)=0$ for the last interval $b$.

911 We tested Equation 4 on two different distributions of fitness effects (Figure 7 and 912 Supplementary Figure S3). To perform those two tests we did simulations under the Poisson 913 Random Field model using PReFerSim (Ortega-Del Vecchyo et al. 2016) to estimate $914 P_{\psi}\left(f \mid \boldsymbol{s}_{\boldsymbol{j}}, D\right)$. We did those simulations using the mouse distribution of fitness effects (Halligan et 915 al. 2013) and the population expansion demographic model. Those calculations were done 916 across 5,000 simulation replicates where the value of $\Theta / 2$ in the first epoch was set equal to 917 1,000. We sampled 4,000 chromosomes for each segregating site to calculate $f$.

918 When we estimated the distribution of fitness effects of new variants in the UK10K data, 919 we estimated $P_{\psi}\left(f \mid \boldsymbol{s}_{\boldsymbol{j}}, D\right)$ by performing 1,000 replicate simulations under the inferred UK10K 920 demographic model and the human distribution of fitness effects (Boyko et al. 2008). The value 921 of $\Theta / 2$ in the first epoch of each simulation was set equal to 1,000. To mimic the properties of 922 the UK10K data, we sampled 7,242 chromosomes for each segregating site. We calculated 
$923 P_{\psi}\left(f \mid \boldsymbol{s}_{\boldsymbol{j}}, D\right)$ by counting the proportion of variants in our 1,000 simulations that have a frequency

$924 f$ equal to $1 \% \pm 0.05 \%$.

925

Estimating $L$ taking into account differences in local recombination rates in the UK10K dataset

Apart from being dependent on the strength of selection acting on the variants, the distribution

930 of $L$ surrounding each variant on the genome in the UK10K data is dependent on the local

931 recombination rate $\rho$. We took into account the local recombination rate when inferring the

932 distribution of fitness effects using the 273 nonCpG nonsynonymous $1 \%$ frequency variants. To

933 do this, we used our importance sampling method to obtain the distribution of $L$ given the

934 selection coefficient, the inferred demographic scenario, and 21 different recombination rates.

935 To select the 21 recombination rates, we used the results from a previously inferred 936 recombination map (Kong et al. 2010). We took the 21 different percentile values $\left(0^{\text {th }}, 5^{\text {th }}, \ldots, 95^{\text {th }}\right.$,

$\left.937100^{\text {th }}\right)$ from the distribution of 546 average recombination rates per base taken from the 938 upstream and downstream $250 \mathrm{~kb}$ regions next to the 273 nonsynonymous $1 \%$ frequency 939 variants. In the end, we generated 21 distributions for each selection value explored, each with 940 a different recombination rate $\rho_{j}$. Those 21 distributions of $\mathcal{L}\left(4 N s, f, D, \rho_{j} \mid L \in w_{i}\right)$ were used to

941 infer selection using the upstream and downstream regions from the nonCpG nonsynonymous $9421 \%$ frequency variants. They were also used to infer the point estimate of $4 N s$ in the nonCpG 943 synonymous $1 \%$ frequency variants. The $\mathcal{L}\left(4 N s, f, D, \rho_{j} \mid L \in w_{i}\right)$ distribution used for each of the 944546 regions is the one where the local recombination $\rho$ is closer to $\rho_{j}$.

945 We evaluated the accuracy of our method to infer selection under the inferred scaled 946 UK10K demographic scenario using simulations. We mimicked the amount of information 947 present in the UK10K data in each simulation replicate. Each simulation replicate contains 273 948 independent loci with 72 haplotypes containing the derived allele. The recombination rates, both 949 to the left and right side of the loci, were equal to the average per base recombination rates in

950 the $250 \mathrm{~kb}$ windows next to each locus in the data. We calculated $L$ going to the left and right 951 side of the focal loci, obtaining $\left(\begin{array}{c}72 \\ 2\end{array}\right) \times 2 \times 273 L$ values for each simulation replicate 952 (Supplementary Figure S8, S10).

953

954 Data availability

955 
956 The programs and data to reproduce every figure of the paper can be found in

957 https://github.com/dortegadelv/HaplotypeDFEStandingVariation .

958

959

Acknowledgements

961

962 We thank Christian Huber, Bernard Kim, Evan Koch, Charleston Chiang, Tanya Phung, Clare

963 Marsden, Annabel Beichman, Jazlyn Mooney and Ying Zhen for useful discussions. We also

964 thank Jair S. Garcia-Sotelo, Luis Alberto Aguilar Bautista, Alejandra Castillo and Carina Uribe

965 for technical assistance. Infrastructure for computational analysis was in part provided by a

966 CONACYT grant led by Alejandra Medina-Rivera [269449]. This work was supported by the UC

967 MEXUS-CONACYT fellowship 213627 to D.O.-D.V, NIH grant R35GM119856 to K.E.L., and

968 NIH grant R01HG007089 to J.N.

969

970 Bibliography

971

972 Adzhubei IA, Schmidt S, Peshkin L et al. (2010) A method and server for predicting damaging

973 missense mutations. Nature Methods, 7, 248-249.

974 Albers PK, McVean G (2018) Dating genomic variants and shared ancestry in population-scale 975 sequencing data. bioRxiv, 416610.

976 Andolfatto P, Nordborg M (1998) The effect of gene conversion on intralocus associations.

977 Genetics, 148, 1397-1399.

978 Auton A, Abecasis GR, Altshuler DM et al. (2015) A global reference for human genetic 979 variation. Nature, 526, 68-74.

980 Balick DJ, Do R, Cassa CA, Reich D, Sunyaev SR (2015) Dominance of deleterious alleles

981 controls the response to a population bottleneck. PLoS Genetics, 11, e1005436.

982 Bataillon T, Bailey SF (2014) Effects of new mutations on fitness: Insights from models and 983 data. Annals of the New York Academy of Sciences, 1320, 76-92.

984 Beichman AC, Phung TN, Lohmueller KE (2017) Comparison of single genome and allele 985 frequency data reveals discordant demographic histories. G3, 7, 3605-3620.

986 Boyko AR, Williamson SH, Indap AR et al. (2008) Assessing the evolutionary impact of amino 987 acid mutations in the human genome. PLoS Genetics, 4, e1000083.

988 Brandvain Y, Wright SI (2016) The limits of natural selection in a nonequilibrium world. Trends 
in Genetics, 32, 201-210.

Braverman JM, Hudson RR, Kaplan NL, Langley CH, Stephan W (1995) The hitchhiking effect on the site frequency spectrum of DNA populations. Genetics, 140, 783-786.

992 Bustamante CD, Wakeley J, Sawyer S, Hartl DL (2001) Directional selection and the site993 frequency spectrum. Genetics, 159, 1779-1788.

994 Charlesworth D, Charlesworth B, Morgan MT (1995) The pattern of neutral molecular variation 995 under the background selection model. Genetics, 141, 1619-32.

996 Charlesworth J, Eyre-Walker A (2007) The other side of the nearly neutral theory, evidence of slightly advantageous back-mutations. Proceedings of the National Academy of Sciences of the United States of America, 104, 16992-7.

Charlesworth B, Morgan MT, Charlesworth D (1993) The effect of deleterious mutations on neutral molecular variation. Genetics, 134, 1289-303.

Chen H, Hey J, Slatkin M (2015) A hidden markov model for investigating recent positive selection through haplotype structure. Theoretical Population Biology, 99, 18-30.

Chen H, Slatkin M (2013) Inferring selection intensity and allele age from multi-locus haplotype structure. G3, 3, 1429-1442.

1006 Population Biology, 66, 219-232.

1007 Crow JF (1972) The dilemma of nearly neutral mutations: how important are they for evolution 1008 and human welfare? Journal of Heredity, 63, 306-316.

1009 Cutter AD, Payseur B a (2013) Genomic signatures of selection at linked sites: unifying the 1010 disparity among species. Nature Reviews Genetics, 14, 262-74.

1011 Cvijović I, Good BH, Desai MM (2018) The effect of strong purifying selection on genetic 1012 diversity. Genetics, 209, 1235-1278.

1013 Delaneau O, Howie B, Cox AJ, Zagury JF, Marchini J (2013a) Haplotype estimation using 1014 sequencing reads. American Journal of Human Genetics, 93, 687-696.

1015 Delaneau O, Zagury JF, Marchini J (2013b) Improved whole-chromosome phasing for disease 1016 and population genetic studies. Nature Methods, 10, 5-6.

1017 Dickinson WJ (2008) Synergistic fitness interactions and a high frequency of beneficial changes 1018 among mutations accumulated under relaxed selection in Saccharomyces cerevisiae.

1019 Genetics, 178, 1571-1578.

1020 Do R, Balick D, Li H et al. (2015) No evidence that selection has been less effective at removing deleterious mutations in Europeans than in Africans. Nature Genetics, 47, 126-131.

1022 Domingo-Calap P, Cuevas JM, Sanjuán R (2009) The fitness effects of random mutations in 
single-stranded DNA and RNA bacteriophages. PLoS Genetics, 5, 1-7.

Eyre-Walker A (2010) Genetic architecture of a complex trait and its implications for fitness and genome-wide association studies. Proceedings of the National Academy of Sciences of the United States of America, 107 (1), 1752-1756.

Eyre-Walker A, Woolfit M, Phelps T (2006) The distribution of fitness effects of new deleterious amino acid mutations in humans. Genetics, 173, 891-900.

Fearnhead P, Donnelly P (2001) Estimating recombination rates from population genetic data. Genetics, 159, 1299-1318.

Ferrer-Admetlla A, Liang M, Korneliussen T, Nielsen R (2014) On detecting incomplete soft or hard selective sweeps using haplotype structure. Molecular Biology and Evolution, 31, $1275-1291$.

Field Y, Boyle EA, Telis N, Gao Z, Gaulton KJ (2016) Detection of human adaptation during the past 2,000 years. Science, 354, 760-764.

Fortier AL, Coffman AJ, Struck TJ et al. (2019) DFEnitely different: Genome-wide

Francioli LC, Menelaou A, Pulit SL et al. (2014) Whole-genome sequence variation, population structure and demographic history of the Dutch population. Nature Genetics, 46, 818.

Galtier N (2016) Adaptive protein evolution in animals and the effective population size

1041 hypothesis. PLoS Genetics, 12, 1-23.

1042 Garud NR, Messer PW, Buzbas EO, Petrov DA (2015) Recent selective sweeps in North

1043 American Drosophila melanogaster show signatures of soft sweeps. PLoS Genetics, 11, 1104432.

1045 Gazave E, Chang D, Clark AG, Keinan A (2013) Population growth inflates the per-individual 1046 number of deleterious mutations and reduces their mean effect. Genetics, 195, 969-978. 1047 Gelman A, Carlin JB, Stern HS et al. (2013) Bayesian Data Analysis. CRC Press, Boca Raton, $1048 \quad$ Florida.

1049 Gossmann TI, Keightley PD, Eyre-Walker A (2012) The effect of variation in the effective 1050 population size on the rate of adaptive molecular evolution in eukaryotes. Genome Biology 1051 and Evolution, 4, 658-667.

1052 Gravel S (2016) When is selection effective. Genetics, 203, 451-462.

1053 Gulko B, Hubisz MJ, Gronau I, Siepel A (2015) A method for calculating probabilities of fitness 1054 consequences for point mutations across the human genome. Nature Genetics, 47, 2761055283.

1056 Gutenkunst RN, Hernandez RD, Williamson SH, Bustamante CD (2009) Inferring the joint 
demographic history of multiple populations from multidimensional SNP frequency data.

PLoS Genetics, 5, e1000695.

Haller BC, Galloway J, Kelleher J, Messer PW, Ralph PL (2019) Tree-sequence recording in SLiM opens new horizons for forward-time simulation of whole genomes. Molecular Ecology Resources, 19, 552-566.

Halligan DL, Keightley PD (2009) Spontaneous Mutation Accumulation Studies in Evolutionary Genetics. Annual Review of Ecology, Evolution, and Systematics, 40, 151-172.

Halligan DL, Kousathanas A, Ness RW et al. (2013) Contributions of protein-coding and regulatory change to adaptive molecular evolution in murid rodents. PLoS Genetics, 9, e1003995.

Harris K, Nielsen R (2013) Inferring demographic history from a spectrum of shared haplotype lengths. PLoS Genetics, 9.

Hartl DL, Moriyama EN, Sawyer SA (1994) Selection intensity for codon bias. Genetics, 138, 227-234.

Henn BM, Botigué LR, Bustamante CD, Clark AG, Gravel S (2015) Estimating the mutation load in human genomes. Proceedings of the National Academy of Sciences, 16, 1-11.

Hietpas RT, Jensen JD, Bolon DNA (2011) Experimental illumination of a fitness landscape.

Hsieh PH, Veeramah KR, Lachance J et al. (2016) Whole-genome sequence analyses of 290.

Huang Y-F, Siepel A (2019) Estimation of allele-specific fitness effects across human proteincoding sequences and implications for disease. Genome Research, gr.245522.118.

Huber CD, DeGiorgio M, Hellmann I, Nielsen R (2016) Detecting recent selective sweeps while controlling for mutation rate and background selection. Molecular Ecology, 25, 142-156.

Huber CD, Kim BY, Marsden CD, Lohmueller KE (2017) Determining the factors driving selective effects of new nonsynonymous mutations. Proceedings of the National Academy of Sciences, 114, 4465-4470.

Hudson RR, Kaplan NL (1995) Deleterious background selection with recombination. Genetics, 141, 1605-1617.

Jacquier H, Birgy A, Le Nagard H et al. (2013) Capturing the mutational landscape of the betalactamase TEM-1. Proceedings of the National Academy of Sciences, 110, 13067-13072. Kaplan NL, Hudson RR, Langley CH (1989) The "hitchhiking effect" revisited. Genetics, 123, 
887-99.

1092 Karolchik D, Baertsch R, Diekhans M et al. (2003) The UCSC Genome Browser Database. Nucleic Acids Research, 31, 51-54.

Keightley PD, Eyre-Walker A (2007) Joint inference of the distribution of fitness effects of deleterious mutations and population demography based on nucleotide polymorphism frequencies. Genetics, 177, 2251-2261.

Kelleher J, Thornton KR, Ashander J, Ralph PL (2018) Efficient pedigree recording for fast population genetics simulation. PLoS Computational Biology, 248500.

Kiezun A, Pulit SL, Francioli LC et al. (2013) Deleterious alleles in the human genome are on average younger than neutral alleles of the same frequency. PLoS Genetics, 9, e1003301.

Kim BY, Huber CD, Lohmueller KE (2017) Inference of the distribution of selection coefficients for new nonsynonymous mutations using large samples. Genetics, 206, 345-361.

Kim Y, Stephan W (2002) Detecting a local signature of genetic hitchhiking along a recombining

Kimura M, Crow JF (1964) The Number of alleles that can be maintained in a finite population.

1107 Kircher M, Witten DM, Jain P et al. (2014) A general framework for estimating the relative 1108 pathogenicity of human genetic variants. Nature Genetics, 46, 310-315.

1109 Koch E, Novembre J (2017) A temporal perspective on the interplay of demography and 1110 selection on deleterious variation in humans. G3, 7, g3.117.039651.

1111 Kong A, Thorleifsson G, Gudbjartsson DF et al. (2010) Fine-scale recombination rate

1112 differences between sexes, populations and individuals. Nature, 467, 1099-103.

1113 Korunes KL, Noor MAF (2017) Gene conversion and linkage: effects on genome evolution and 1114 speciation. Molecular Ecology, 26, 351-364.

1115 Kousathanas A, Keightley PD (2013) A comparison of models to infer the distribution of fitness 1116 effects of new mutations. Genetics, 193, 1197-1208.

1117 Li H (2011) A new test for detecting recent positive selection that is free from the confounding 1118 impacts of demography. Molecular Biology and Evolution, 28, 365-375.

1119 Li Y, Vinckenbosch N, Tian G et al. (2010) Resequencing of 200 human exomes identifies an 1120 excess of low-frequency non-synonymous coding variants. Nature Genetics, 42, 969-972.

1121 Lind PA, Berg OG, Andersson DI (2010) Mutational robustness of ribosomal protein genes. 1122 Science, 330, 825-827.

1123 Lohmueller KE (2014a) The impact of population demography and selection on the genetic 1124 architecture of complex traits. PLoS Genetics, 10. 
1125 Lohmueller KE (2014b) The distribution of deleterious genetic variation in human populations.

1126 Current Opinion in Genetics and Development, 29, 139-146.

1127 Lynch M (2016) Mutation and human exceptionalism: Our future genetic load. Genetics, 202, 1128 869-875.

Mancuso N, Rohland N, Tandon A et al. (2015) The contribution of rare variation to prostate cancer heritability. Nature Genetics, 48, 30-35.

Marsden CD, Ortega-Del Vecchyo D, O’Brien DP et al. (2016) Bottlenecks and selective sweeps during domestication have increased deleterious genetic variation in dogs. Proceedings of the National Academy of Sciences, 113, 152-157.

Martin G, Lenormand T (2006) A general multivariate extension of Fisher's geometrical model and the distribution of mutation fitness effects across species. Evolution, 60, 893-907.

Maruyama T (1974) The age of an allele in a finite population. Genetical research, 23, 137-143. e1004528.

Maynard Smith J, Haigh J (1974) The hitch-hiking effect of a favourable gene. Genetical 1140 research, 23, 23-35.

1141 McCarthy S, Das S, Kretzschmar W et al. (2016) A reference panel of 64,976 haplotypes for 1142 genotype imputation. Nature Genetics, 48, 1279-1283.

1143 McVicker G, Gordon D, Davis C, Green P (2009) Widespread genomic signatures of natural 1144 selection in hominid evolution. PLoS genetics, 5, e1000471.

1145 Nadachowska-Brzyska K, Burri R, Smeds L, Ellegren H (2016) PSMC analysis of effective 1146 population sizes in molecular ecology and its application to black-and-white Ficedula 1147 flycatchers. Molecular Ecology, 25, 1058-1072.

1148 Nakagome S, Alkorta-Aranburu G, Amato R et al. (2016) Estimating the ages of selection 1149 signals from different epochs in human history. Molecular Biology and Evolution, 33, 6571150669.

1151 Nicolaisen LE, Desai MM (2013) Distortions in genealogies due to purifying selection and 1152 recombination. Genetics, 195, 221-230.

1153 Nielsen R (2005) Molecular signatures of natural selection. Annual Review of Genetics, 39, 1154 197-218.

1155 Nordborg M, Charlesworth B, Charlesworth D (1996) The effect of recombination on 1156 background selection. Genetical research, 67, 159-174.

1157 Ohta T (1992) The nearly neutral theory of molecular evolution. Annual Review of Ecology and 1158 Systematics, 23, 263-286. 
1159 Ormond L, Foll M, Ewing GB, Pfeifer SP, Jensen JD (2016) Inferring the age of a fixed

1160 beneficial allele. Molecular Ecology, 25, 157-169.

1161 Ortega-Del Vecchyo D, Marsden CD, Lohmueller KE (2016) PReFerSim: fast simulation of 1162 demography and selection under the Poisson Random Field model. Bioinformatics, 32 , 1163 3516-3518.

1164 Pavlidis P, Jensen JD, Stephan W (2010) Searching for footprints of positive selection in whole1165 genome SNP data from nonequilibrium populations. Genetics, 185, 907-922.

1166 Peischl S, Dupanloup I, Kirkpatrick M, Excoffier L (2013) On the accumulation of deleterious 1167 mutations during range expansions. Molecular Ecology, 22, 5972-5982.

1168 Peris JB, Davis P, Cuevas JM, Nebot MR, Sanjuán R (2010) Distribution of fitness effects 1169 caused by single-nucleotide substitutions in bacteriophage f1. Genetics, 185, 603-609.

1170 Platt A, Hey J (2017) Recent African gene flow responsible for excess of old rare genetic 1171 variation in Great Britain. bioRxiv.

1172 Przeworski M (2003) Estimating the time since the fixation of a beneficial allele. Genetics, 164, 1173 1667-1676.

1174 Racimo F, Schraiber JG (2014) Approximation to the distribution of fitness effects across 1175 functional categories in human segregating polymorphisms. PLoS Genetics, 10, e1004697. 1176 Ragsdale AP, Coffman AJ, Hsieh P, Struck TJ, Gutenkunst RN (2016) Triallelic population 1177 genomics for inferring correlated fitness effects of same site nonsynonymous mutations. 1178 Genetics, 203, 513-523.

1179 Rice DP, Good BH, Desai MM (2015) The evolutionarily stable distribution of fitness effects. $1180 \quad$ Genetics, 200, 321-329.

1181 Robert CP, Casella G (2010) Introducing Monte Carlo Methods with R. Springer.

1182 Sabeti PC, Reich DE, Higgins JM et al. (2002) Detecting recent positive selection in the human 1183 genome from haplotype structure. Nature, 419, 832-837.

1184 Sabeti PC, Varilly P, Fry B et al. (2007) Genome-wide detection and characterization of positive 1185 selection in human populations. Nature, 449, 913-8.

1186 Sanjuán R, Moya A, Elena SF (2004) The distribution of fitness effects caused by single1187 nucleotide substitutions in an RNA virus. Proceedings of the National Academy of Sciences 1188 of the United States of America, 101, 8396-8401.

1189 Sawyer SA, Hartl DL (1992) Population genetics of polymorphism and divergence. Genetics, $1190 \quad 132,1161-1176$.

1191 Schraiber JG, Griffiths RC, Evans SN (2013) Analysis and rejection sampling of Wright-Fisher 1192 diffusion bridges. Theoretical Population Biology, 89, 64-74. 
1193 Ségurel L, Wyman MJ, Przeworski M (2014) Determinants of mutation rate variation in the human germline. Annual Review of Genomics and Human Genetics, 15, 47-70.

Serohijos AWR, Shakhnovich El (2014) Contribution of selection for protein folding stability in shaping the patterns of polymorphisms in coding regions. Molecular Biology and Evolution, 31, 165-176.

Sim NL, Kumar P, Hu J et al. (2012) SIFT web server: Predicting effects of amino acid substitutions on proteins. Nucleic Acids Research, 40, 452-457.

Simons YB, Sella G (2016) The impact of recent population history on the deleterious mutation load in humans and close evolutionary relatives. Current Opinion in Genetics and Development, 41, 150-158.

Simons YB, Turchin MC, Pritchard JK, Sella G (2014) The deleterious mutation load is insensitive to recent population history. Nature Genetics, 46, 220-4.

Slatkin M (2001) Simulating genealogies of selected alleles in a population of variable size. Genetical Research, 78, 49-57.

Slatkin M (2008) A Bayesian method for jointly estimating allele age and selection intensity.

1208 Genetics Research, 90, 129-137.

1209 Slatkin M, Rannala B (1997) Estimating the age of alleles by use of intraallelic variability.

1210 American Journal of Human Genetics, 60, 447-58.

1211 Smith J, Coop G, Stephens M, Novembre J (2018) Estimating time to the common ancestor for 1212 a beneficial allele. Molecular Biology and Evolution, 35, 1003-1017.

1213 Somel M, Sayres MAW, Jordan G et al. (2013) A scan for human-specific relaxation of negative 1214 selection reveals unexpected polymorphism in proteasome genes. Molecular Biology and 1215 Evolution, 30, 1808-1815.

1216 Song S, Sliwerska E, Emery S, Kidd JM (2017) Modeling human population separation history 1217 using physically phased genomes. Genetics, 205, 385-395.

1218 Tang K, Thornton KR, Stoneking M (2007) A new approach for using genome scans to detect 1219 recent positive selection in the human genome. PLoS Biology, 5, 1587-1602.

1220 Tataru P, Mollion M, Glemin S, Bataillon T (2017) Inference of distribution of fitness effects and 1221 proportion of adaptive substitutions from polymorphism data. Genetics, 207, 1103-119.

1222 Tenaillon O (2014) The utility of Fisher's geometric model in evolutionary genetics. Annual 1223 Review of Ecology, Evolution, and Systematics, 45, 179-201.

1224 Tishkoff SA, Reed FA, Ranciaro A et al. (2007) Convergent adaptation of human lactase 1225 persistence in Africa and Europe. Nature Genetics, 39, 31-40.

1226 Voight BF, Kudaravalli S, Wen XQ, Pritchard JK (2006) A map of recent positive selection in the 
human genome. PLoS Biology, 4, 446-458.

Walter K, Min JL, Huang J et al. (2015) The UK10K project identifies rare variants in health and disease. Nature, 526, 82-90.

Wang ET, Kodama G, Baldi P, Moyzis RK (2006) Global landscape of recent inferred darwinian selection for Homo sapiens. Proceedings of the National Academy of Sciences, 103, 135140.

Williams AL, Genovese G, Dyer T et al. (2015) Non-crossover gene conversions show strong GC bias and unexpected clustering in humans. eLife, 4, 1-21. and population growth from patterns of variation in the human genome. Proceedings of the National Academy of Sciences, 102, 7882-7887.

Williamson SH, Hubisz MJ, Clark AG et al. (2007) Localizing recent adaptive evolution in the human genome. PLoS Genetics, 3, 0901-0915. 